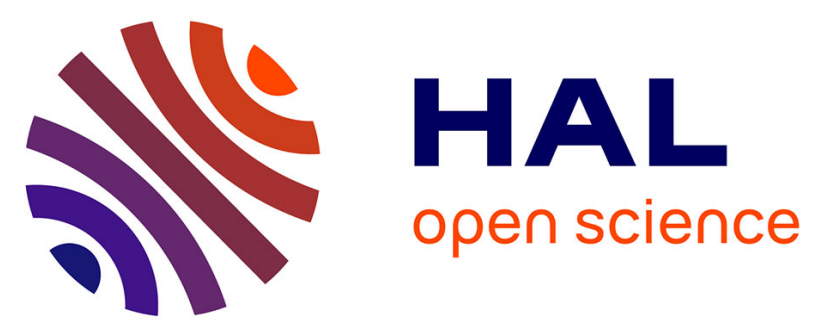

\title{
Strong Neotectonic Block Rotations, Related to the Africa-Eurasia Convergence in Northern Algeria: Paleomagnetic Evidence From the Mitidja Basin
}

M. E. M. Derder, H. Djellit, B. Henry, S. Maouche, M. Amenna, R. Bestandji, H. Ymel, S. Gharbi, A. Abtout, C. Dorbath

\section{- To cite this version:}

M. E. M. Derder, H. Djellit, B. Henry, S. Maouche, M. Amenna, et al.. Strong Neotectonic Block Rotations, Related to the Africa-Eurasia Convergence in Northern Algeria: Paleomagnetic Evidence From the Mitidja Basin. Tectonics, 2019, 38, pp.4249-4266. 10.1029/2018TC005394 . insu-03586585

\section{HAL Id: insu-03586585 \\ https://hal-insu.archives-ouvertes.fr/insu-03586585}

Submitted on 24 Feb 2022

HAL is a multi-disciplinary open access archive for the deposit and dissemination of scientific research documents, whether they are published or not. The documents may come from teaching and research institutions in France or abroad, or from public or private research centers.
L'archive ouverte pluridisciplinaire HAL, est destinée au dépôt et à la diffusion de documents scientifiques de niveau recherche, publiés ou non, émanant des établissements d'enseignement et de recherche français ou étrangers, des laboratoires publics ou privés.

$$
\text { Copyright }
$$




\section{Tectonics}

\section{RESEARCH ARTICLE \\ 10.1029/2018TC005394}

\section{Special Section:}

Geodynamics, Crustal and Lithospheric Tectonics, and active deformation in the Mediterranean Regions (A tribute to Prof. Renato

Funiciello)

Key Points:

- Paleomagnetism shows that Mitidja basin was affected since 16 Ma by clockwise rotations, coherent in average of $48^{\circ}$ for the largest blocks

- Along shearing structures, smaller blocks show various rotations, in some cases of large magnitude

- These rotations are interpreted as resulting from bookshelf neotectonics, consequence of the Africa-Eurasia plates convergence

Correspondence to:

M. E. M. Derder,

m.e.m.derder@gmail.com

Citation:

Derder, M. E. M., Djellit, H., Henry, B. Maouche, S., Amenna, M., Bestandji,

R., et al (2019). Strong neotectonic block-rotations, related to the

Africa-Eurasia convergence in northern Algeria: Paleomagnetic evidence from the Mitidja basin. Tectonics, 38 , 4249-4266. https://doi.org/ 10.1029/2018TC005394

Received 3 NOV 2018 Accepted 21 SEP 2019 Published online 16 DEC 2019

(C)2019. American Geophysical Union. All Rights Reserved.

\section{Strong Neotectonic Block-Rotations, Related to the Africa- Eurasia Convergence in Northern Algeria: Paleomagnetic Evidence From the Mitidja Basin}

\author{
M.E.M. Derder ${ }^{1}$ (D) H. Djellit ${ }^{1}$, B. Henry ${ }^{2}$, S. Maouche ${ }^{1}$, M. Amenna ${ }^{1}$, R. Bestandji ${ }^{1}$, H. Ymel ${ }^{1}$, \\ S. Gharbi ${ }^{1}$, A. Abtout ${ }^{1}$, and C. Dorbath ${ }^{3}$ \\ ${ }^{1}$ C.R.A.A.G., Bouzaréah, Alger, Algeria, ${ }^{2}$ Paléomagnétisme, Institut de Physique du Globe de Paris, Sorbonne Paris Cité, \\ Univ Paris and UMR 7154 CNRS, 4 avenue de Neptune, Saint-Maur, France, ${ }^{3}$ Sismologie, École et Observatoire des \\ Sciences de la Terre, Univ. Strasbourg and UMR 7516 CNRS, 5 rue René Descartes, Strasbourg cedex, France
}

\begin{abstract}
Significant tectonic clockwise rotations were evidenced in the Tell Atlas (Neogene Algerian Cheliff basin) by previous paleomagnetic studies. For northwestern Africa and in the context of the AfricaEurasia convergence, they provided a new argument validating a kinematic model, based on transpression with shortening accommodated by clockwise block rotations. To corroborate this deformation pattern at a larger scale, new paleomagnetic studies were performed on 349 cores in the Mitidja basin, of Mio-PlioQuaternary age, in the Algerian Tell Atlas. This intramontaneous basin is structured by two regional major EW to WNW-ESE dextral shear zones. Primary magnetization data were obtained in 43 out of the 49 sampled sites. This magnetization is carried by Ti-poor titanomagnetite. Its direction shows that significant tectonic block rotations affected this basin since $16 \mathrm{Ma}$. Zones located between the E-W and WNW-ESE major structures are affected by coherent clockwise rotations (average magnitude of $48^{\circ}$ ) of large blocks, compartmented by presently associated sinistral NE-SW faults. Along the shearing structures, smaller blocks, resulting from the fragmentation of the large blocks, show various rotations, many of which are of large magnitude. These rotations, similar to those highlighted previously in the Cheliff basin, are interpreted as resulting from bookshelf, consequence of the Africa-Eurasia plates convergence in the Tell Atlas.
\end{abstract}

\section{Introduction}

It is well known that the crustal deformation in the North African margin is related to the Africa-Eurasia plate convergence. This convergence began during the Late Cretaceous (Dewey et al., 1989; Le Pichon et al., 1988; Ricou, 1994) and pursues up to the present time, causing seismic activity, particularly along the Africa-Eurasia plate boundary in the Tell Atlas of Algeria (see Meghraoui, 2018, and references herein). The Tell Atlas experienced large and destructive earthquakes, for example, the 10 October 1980, El-Asnam Ms 7.3 (Ouyed et al., 1981) and the 21 May 2003 Zemmouri Mw 6.8 (Ayadi et al., 2003) earthquakes being among the largest recent seismic events recorded in the western Mediterranean.

A counterclockwise rotation of Africa with respect to Eurasia since Late Cretaceous was established from magnetic anomaly studies in the Atlantic Ocean (Dewey et al., 1989; Mazzoli \& Helman, 1994; Rosenbaum et al., 2002, and references therein). The oblique plate convergence resulting from this counterclockwise rotation of Africa created a tectonic transpression with N-S to NNW-SSE shortening direction (Mauffret, 2007; Morel \& Meghraoui, 1996).

Geodetic models using GPS (Nocquet, 2012; Nocquet \& Calais, 2004; Serpelloni et al., 2007) support AfricaEurasia plate convergence as well as the associated transpression tectonic system for the recent times. The stress inversion of the largest-magnitude earthquake focal mechanisms in the western Tell Atlas shows a compressive tectonic regime $\left(R 0=2.43 \pm 0.21\right.$, with $\mathrm{SHmax} N 147^{\circ} \mathrm{E} \pm 9.4^{\circ}$; Soumaya et al., 2018). Moreover, the focal mechanism solutions of almost all the earthquakes (Ousadou et al., 2014; Soumaya et al., 2018) provide NW and NE trending focal planes suggesting their reactivation and role in directional control of the regional tectonic pattern. These main tectonic structures confirm that this segment of the plate boundary is a transpressive deformed margin, where active NW trending dextral and NE reverse and sinistral faults are controlled by a first-order E-W right-lateral transcurrent fault system of the Western Tell (Meghraoui et al., 1996; Meghraoui \& Pondrelli, 2012). 


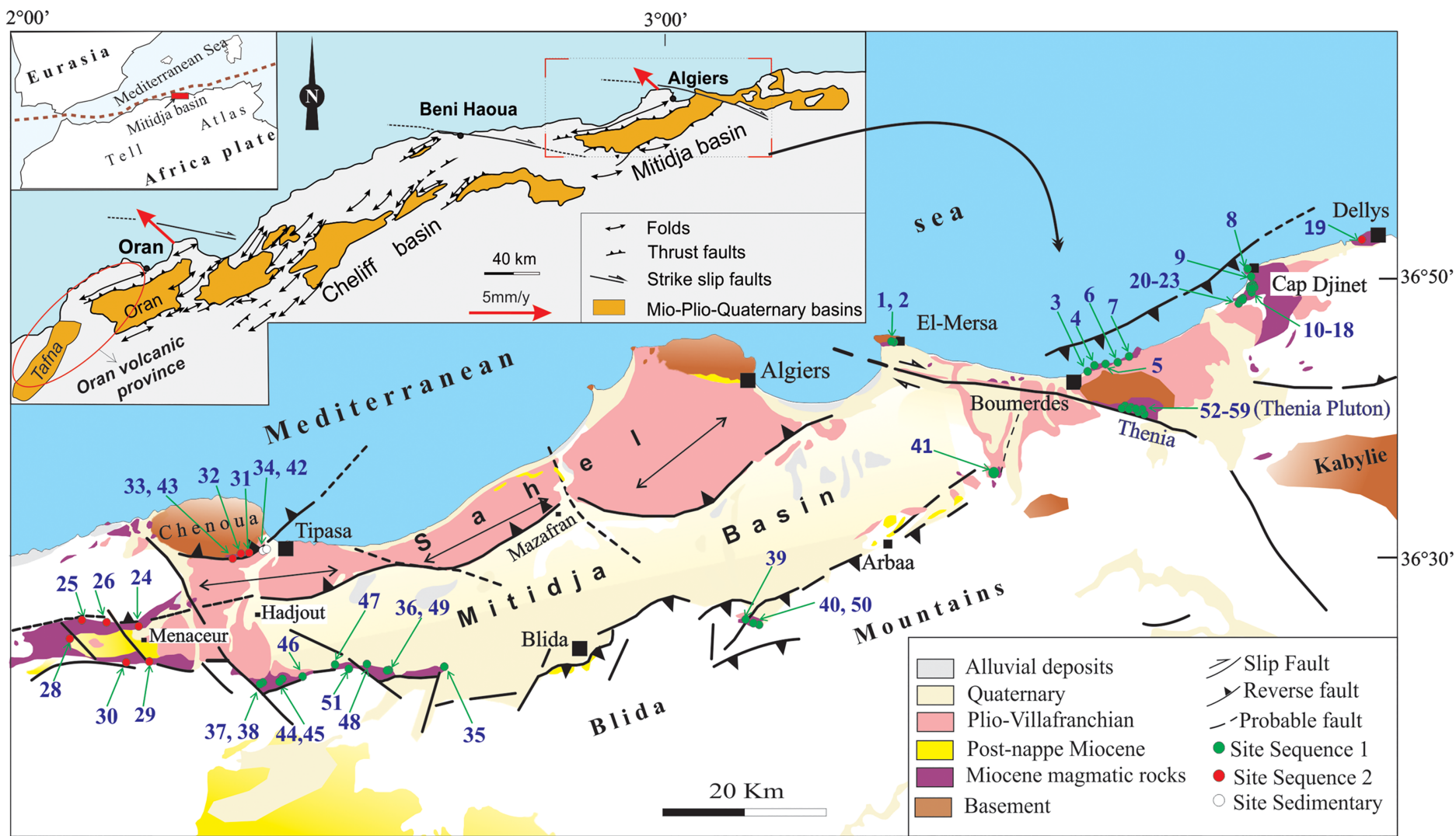

Figure 1. Geological map of the Mitidja basin and location of the sampling sites. Sequence 1 between 16 and 15 Ma and Sequence 2 between 13 and 11 Ma. Red arrows show the velocity field in a Eurasia fixed reference frame (Nocquet, 2012).

Based on structural and seismological data, it was suggested that the NE-SW left-lateral and the E-W rightlateral faults can accommodate the NNE-SSW convergence through clockwise rotation of large blocks (Meghraoui \& Pondrelli, 2012). Thomas (1985), Meghraoui et al. (1986), and Mauffret et al. (1987) highlighted NE-SW trending active fault-related folds upon E-W trending deep-seated dextral faults in the Tell Atlas and along its northern margin (see Figure 1 in Meghraoui \& Pondrelli, 2012). This tectonic system favored the division of northern Algeria into several NE-SW-oriented tectonics blocks. Regarding the NNW-SSE transpression of plate convergence, the tectonic context must infer block rotations as suggested by Meghraoui et al. (1996) and Meghraoui and Pondrelli (2012) in a "bookshelf" kinematic model. The deformation pattern, involving clockwise block rotations in northern Algeria, is supported by the paleomagnetic results obtained on Late Cenozoic rocks in the Chellif basin (Aïfa et al., 1992; Derder et al., 2011, 2013) and Oran volcanic provinces (Figure 1), in the vicinity of Oran and Tafna basins (Derder et al., 2018). These previous works pointed out tectonic rotations from large to small size blocks.

This paper addresses the quantification of the block rotations in relation with the movements along the main faults crossing the Mitidja basin adjacent to the Chellif basin (Figure 1). This should allow construction and assessment of the dynamic evolution of the North Africa border in the context of the Africa-Eurasia active plate convergence. Miocene volcanic rocks have been mainly selected for a paleomagnetic study that represents an efficient and reliable approach to highlight eventual tectonic rotations. Magnetic fabric of the "Thenia" Miocene granodiorite (Figure 1), intruded in a key position along one of the major faults affecting the basin, has been analyzed in order to obtain information about the stress field during the pluton emplacement.

\section{Geological Setting}

The Tell Atlas thrust belt results from the plate convergence (present rate about 4 to $6 \mathrm{~mm} / \mathrm{yr}$ ) of Africa toward Eurasia (Nocquet, 2012; Nocquet \& Calais, 2004; Serpelloni et al., 2007). The Algerian Mitidja basin (Figure 1), one of the most seismically active area in northern Africa, belongs to the central Tell Atlas. It is E- 
W to ENE-WSW trending, over $150 \mathrm{~km}$, being more or less parallel to the coastline, and is associated with Quaternary compressive deformation revealed by thrust focal mechanisms of significant earthquakes (Maouche et al., 2011; Meghraoui, 1991). The northern part of this basin shows the Sahel anticline (Figure 1) related to a blind or hidden reverse fault probably at the origin of several earthquakes that occurred in the vicinity of Algiers (Maouche et al., 2011; Meghraoui, 1991). Its main part is a trough filled by Miocene and Plio-Quaternary sediments. Its southern boundary (Figure 1) corresponds to an E-W to NE-SW faults system (referred here as the Blida faults system) that is marked by geomorphic scarps with up to 1,000 m vertical drop (Guemache, 2010; Maouche et al., 2011; Meghraoui, 1988).

In the Mitidja basin (Guemache, 2010; Maouche et al., 2011; Meghraoui, 1991), as in the Chellif basin located farther southwest (Meghraoui \& Doumaz, 1996), the active and neotectonic deformation is characterized by E-W to NE-SW trending fold structures and related reverse and thrust faults, accommodating 2 to $3 \mathrm{~mm} / \mathrm{yr}$ shortening across the Tell Atlas. NE-SW to E-W trending structures, namely, the Blida reverse faults system and associated folds and the Sahel anticline, form the southern and northern edges of the basin, respectively (Figure 1). South of the basin, the Blida Mountains correspond to Paleozoic granites and micaschists, Mesozoic flysch and limestones, and Cenozoic sandy marls, sandstones, and calcarenites (Durand, 1969). The NE offshore continuation of the Blida faults system was reactivated during the 2003 Zemmouri (Mw = 6.8) earthquake (Ayadi et al., 2003; Meghraoui et al., 2004). In the northern part of the Mitidja basin (Figure 1), the ENE-WSW-trending and 70-km-long Sahel asymmetrical anticline shows thrust and flexural faulting, affecting the Neogene and Quaternary units with the bedding plane tilted up to vertical on its southern flank (Maouche et al., 2011; Meghraoui, 1991). Numerous studies published in the framework of MARADJA and SPIRAL projects highlight that offshore Algeria displays active tectonic structures. Leprêtre et al. (2013) discussed the Plio-Quaternary deformation related to the compressional reactivation of the central Algerian Neogene margin as diffuse nature. Domzig et al. (2006) using detailed bathymetry data identified large Neogene reverse faults and folds considered as active. Ratzov et al. (2015) interpreted a turbidites deposits group as resulting from a coseismic movement. The Tell intramountain basins (e.g., Tafna, Oran, Chellif, Mitidja; Figure 1) are often marked by volcanic activity particularly during the opening and evolution period. The Mitidja basin is characterized by the presence of magmatic rocks formations, ranging from 16 to 9 Ma old (Ait-Hamou, 1987; Belanteur, 2001; Lepvrier et al., 1970). Petrographic and geochronologic studies show that rhyolites were first emplaced around 16 to $15 \mathrm{Ma}$, followed by andesites at 13-11 Ma and dacite around 9 Ma (Ait-Hamou, 1987; Belanteur et al., 1995; Bellon et al., 1977; Hernandez \& Lepvrier, 1979). In several places, the volcanic rocks are intercalated within the Miocene sedimentation.

The western Mitidja zone comprises magmatic series ranging from rhyolitic to andesitic rocks indicating typical calco-alcaline petrographic and geochemical characteristics, suggesting, from Bellon and Brousse (1977), Girod and N. Girod (1977), Hernandez et al. (1987), and Belanteur (2001), that these rocks belong to the volcanic activity of the plate boundary. In the eastern sector of the Mitidja basin, Belanteur (2001) describes Miocene tuff beds and igneous rocks in the vicinity of Boumerdes, Thenia, Cap Djinet, and Dellys (Figure 1). These Miocene age rocks appear as steep cliffs along the coast at Dellys. It corresponds to a succession of volcanic flows with variable aspects but with comparable mineral compositions. This series of volcanic flows, separated by volcano-sedimentary deposits, is intercalated in a marly sediment of molasse indicating important neotectonic or post-magmatism tectonic activity.

At the basin scale, volcanic rocks underlie the borders and mark the highly deformed (intense faulting) basin borders. Except very locally, in close contact of faults, they present nonductile internal postmagmatic deformation. In the Hadjout-Menaceur zone (Figure 1), these rocks were affected by folding (E-W trending syncline), highlighting the post-Miocene compressive tectonic activity. In the vicinity of Chenoua massif (Tipasa area) (Figure 1), the volcanic bodies that underlie the northern border of the Mitidja basin and the Sahel fold seem to be developed along the highly deformed zones. As indicated above, this basin constitutes a structurally complex domain with several faults indicating a restraining basin structure linked to transpressive tectonic along a major E-W crustal structure (Mauffret, 2007; Meghraoui, 1988; Meghraoui \& Pondrelli, 2012). Several faults crossing the basin (e.g., the Thenia and Mazafran faults in Figure 1) separate structural compartments (Maouche \& Harbi, 2018). NE-SW faults, with visible motion in the basin southern border, are hidden by sedimentary deposits; they could also separate tectonic blocks (Figure 1). 


\section{Paleomagnetic Sampling and Analytical Procedure}

A paleomagnetic study of favorable exposures of accessible Miocene volcanic sequences was performed in the Mitidja basin in an attempt to determine the magnitude, direction, and the spatial distribution of block displacements and/or rotations that may have accompanied or followed the crustal deformation (Figure 1). Different volcanic sequences in the Mitidja basin are represented by rhyolite, andesite, and basalt sequences corresponding to the two groups of age (16-15 and 13-11 Ma) determined from isotopic dating (Ait-Hamou, 1987; Belanteur et al., 1995; Bellon et al., 1977; Hernandez \& Lepvrier, 1979).

The studied rocks are outcropping mostly along the Mitidja basin margins (Figure 1). Distributed over 49 sites, these rocks were extensively sampled for the paleomagnetic investigations: 349 cores, oriented with magnetic and sun compasses. Because of high weathering, only two to four samples were collected for testing from three "volcanic" outcrops (Sites 1, 2, and 7). Volcanic rocks were sampled in 46 sites (each site corresponding to a single flow). Sites for the oldest group of age are all (except site 19) located in the most western part of the basin (groups of ages of the different sites are indicated in Table 1). Unfortunately, most available outcrops correspond to isolated small bodies, located within or close to faults zones, and are therefore possibly affected by local rotations. Only (i) two quarries with lava piles (Sites 10-18 and 20-23, each site corresponding to a different lava flow), (ii) coastal sites (Sites 3-6), (iii) a site belonging to a large outcrop (Site 8), and (iv) a thick lava flow (Site 28) present in large and continuous outcrops can be considered as representative for determination of a global movement of the basin. The remaining three sites correspond to Pliocene sedimentary formations (Table 1).

One to three specimens of standard size have been cut from each core, allowing pilot specimen demagnetization and additional rock magnetic studies to be accomplished. Before any demagnetization analysis, the specimens were stored in zero magnetic field for at least 1 month to reduce any potential viscous magnetization. The remanent magnetization was measured using a JR5 spinner magnetometer (AGICO, Brno, Czech Republic). Both thermal (TH) and Alternating Field (AF) demagnetizations were performed on pilot specimens, and when required, some other specimens were demagnetized by combined AF-TH procedure. In order to appropriately separate and recognize the magnetization components, numerous demagnetization steps were used. Increments range from 50 to $100{ }^{\circ} \mathrm{C}$ for the lowest temperatures to $10^{\circ} \mathrm{C}$ for the highest ones during $\mathrm{TH}$ demagnetization and from 1 to $10 \mathrm{mT}$ during the AF one (maximum used field intensity: $100 \mathrm{mT}$ ). The results of demagnetization process are presented on orthogonal vector plots (As \& Zijderveld, 1958; Zijderveld, 1967). The remaining magnetization vectors after each step and the difference vectors removed between two consecutive demagnetization steps were plotted on equal area projections. The direction of the magnetization components was calculated by principal component analysis (Kirschvink, 1980). Mean characteristic directions were determined using Fisher's (1953) statistics.

\section{Rock Magnetism}

Representative samples from several sites of volcanic rocks were selected to determine their magnetic mineralogy and characteristic properties from different magnetic methods, based on TH demagnetization curves, thermomagnetic $\mathrm{K}(\mathrm{T})$ curves (low-field susceptibility as a function of temperature), and hysteresis loops. $\mathrm{K}(\mathrm{T})$ curves were measured on AGICO KLY3, CSL-CS3 equipment. Hysteresis loops were mainly obtained from a laboratory-made translation inductometer for small samples (about $3 \mathrm{~cm}^{3}$ ) within an electromagnet, whereas the remaining data were obtained from Micro-Mag VSM equipment.

The Natural Remanent Magnetization has a relatively high intensity, varying from $3.3 \times 10^{-5}$ to $2.2 \mathrm{~A} / \mathrm{m}$, with a mean of $4.3 \times 10^{-1} \mathrm{~A} / \mathrm{m}$. Most TH demagnetization curves show mainly a progressive decrease of the magnetization from $500{ }^{\circ} \mathrm{C}$ up to around $550-580{ }^{\circ} \mathrm{C}$ (Figure 2a, MI300 sample), suggesting the presence of Ti-poor titanomagnetite as magnetic carrier. For some samples, however, this decrease starts at around the $300{ }^{\circ} \mathrm{C}$ (Figure 2a, MI282 sample) step. For some of the samples, all from the basin borders, a weak magnetization decrease continues for temperatures higher than $580{ }^{\circ} \mathrm{C}$ (Figure 2a, MI325 and MI318 samples) suggesting the existence of hematite besides the titanomagnetite. For the weathered samples collected on the coast (Sites 3 to 7), magnetization direction, stable during AF treatment, becomes often erratic from low heating steps $\left(200^{\circ} \mathrm{C}\right)$, suggesting the presence of goethite.

Thermomagnetic curves $\mathrm{K}(\mathrm{T})$ for most sites show a weak increase of magnetic susceptibility up to $\sim 500{ }^{\circ} \mathrm{C}$ (Figure 2b, MI186 and MI283 samples). Partial cooling carried out from these temperature indicates that 
Table 1

Paleomagnetic Results

\begin{tabular}{|c|c|c|c|c|c|c|c|c|c|c|c|c|c|c|c|c|}
\hline Sites & Age & $\lambda$ & $\varphi$ & $\mathrm{n}$ & $\mathrm{n}_{\text {ret }}$ & M & $\mathrm{P}\left({ }^{\circ}\right)$ & $\mathrm{G}\left({ }^{\circ}\right)$ & $\mathrm{D}_{\mathrm{g}}\left({ }^{\circ}\right)$ & $\mathrm{Ig}_{\mathrm{g}}\left({ }^{\circ}\right)$ & $\mathrm{D}_{\mathrm{s}}\left({ }^{\circ}\right)$ & $\mathrm{I}_{\mathrm{S}}\left({ }^{\circ}\right)$ & $\alpha_{95}\left(^{\circ}\right)$ & $\mathrm{k}$ & $\theta\left(^{\circ}\right)$ & $\Delta \theta\left(^{\circ}\right)$ \\
\hline 1 & 2 & $36^{\circ} 48^{\prime} 36.0^{\prime \prime}$ & $3^{\circ} 15^{\prime} 31.1^{\prime \prime}$ & 2 & 0 & 5.4 & 90 & 180 & - & - & - & - & - & - & - & - \\
\hline 2 & 2 & $36^{\circ} 48^{\prime} 36.0^{\prime \prime}$ & $3^{\circ} 15^{\prime} 31.1^{\prime \prime}$ & 2 & 0 & 10.3 & 90 & 180 & - & - & - & - & - & - & - & - \\
\hline 3 & 2 & $36^{\circ} 47^{\prime} 03.4^{\prime \prime}$ & $3^{\circ} 31^{\prime} 10.2^{\prime \prime}$ & 13 & 12 & 0.7 & 30 & 30 & 257.6 & -39.9 & 246.4 & -17.3 & 6.9 & 41 & 65.1 & 7.2 \\
\hline 4 & 2 & $36^{\circ} 47^{\prime} 04.2^{\prime \prime}$ & $3^{\circ} 31^{\prime} 11.8^{\prime \prime}$ & 11 & 5 & 0.3 & 30 & 30 & 292.4 & -58.1 & 257.2 & -44.5 & 3.3 & 527 & 75.9 & 4.6 \\
\hline 5 & 2 & $36^{\circ} 47^{\prime} 06.2^{\prime \prime}$ & $3^{\circ} 31^{\prime} 27.0^{\prime \prime}$ & 7 & 5 & 3.0 & 30 & 30 & 284.6 & -50.6 & 259.0 & -35.8 & 20.4 & 21 & 77.7 & 25.4 \\
\hline 6 & 2 & $36^{\circ} 47^{\prime} 06.4^{\prime \prime}$ & $3^{\circ} 31^{\prime} 28.7^{\prime \prime}$ & 12 & 6 & 0.3 & 30 & 30 & 255.7 & -73.1 & 227.6 & -46.6 & 13.4 & 18 & 46.3 & 19.7 \\
\hline 7 & 2 & $36^{\circ} 47^{\prime} 07.2^{\prime \prime}$ & $3^{\circ} 31^{\prime} 37.7^{\prime \prime}$ & 4 & 0 & 76 & 45 & 330 & - & - & - & - & - & - & - & \\
\hline 8 & 2 & $36^{\circ} 47^{\prime} 07.2^{\prime \prime}$ & $3^{\circ} 31^{\prime} 37.7^{\prime \prime}$ & 8 & 4 & 807 & 35 & 330 & 238.2 & -29.1 & 221.0 & -22.5 & 7.9 & 94 & 39.7 & 8.6 \\
\hline 9 & 2 & $36^{\circ} 52^{\prime} 27.6^{\prime \prime}$ & $3^{\circ} 43^{\prime} 20.2^{\prime \prime}$ & 8 & 6 & 618 & 0 & 0 & 259.7 & -49.6 & 259.7 & -49.6 & 11.3 & 25 & 78.4 & 17.6 \\
\hline 10 & 2 & $36^{\circ} 51^{\prime} 07.4^{\prime \prime}$ & $3^{\circ} 42^{\prime} 34.6^{\prime \prime}$ & 7 & 4 & 854 & 30 & 110 & 223.2 & -20.9 & 230.0 & -7.2 & 4.5 & 294 & 49.4 & 5.8 \\
\hline 11 & 2 & $36^{\circ} 51^{\prime} 24.9^{\prime \prime}$ & $3^{\circ} 42^{\prime} 36.0^{\prime \prime}$ & 6 & 3 & 1900 & 30 & 110 & 218.5 & -20.3 & 225.9 & -8.8 & 5.9 & 131 & & \\
\hline 12 & 2 & $36^{\circ} 51^{\prime} 24.9^{\prime \prime}$ & $3^{\circ} 42^{\prime} 36.0^{\prime \prime}$ & 6 & 5 & 1830 & 30 & 110 & 216.1 & -18.9 & 223.2 & -8.6 & 4.4 & 158 & & \\
\hline 13 & 2 & $36^{\circ} 51^{\prime} 24.9^{\prime \prime}$ & $3^{\circ} 42^{\prime} 36.0^{\prime \prime}$ & 6 & 5 & 748 & 30 & 110 & 219.4 & -18.2 & 225.6 & -6.5 & 6.5 & 142 & & \\
\hline 14 & 2 & $36^{\circ} 51^{\prime} 24.9^{\prime \prime}$ & $3^{\circ} 42^{\prime} 36.0^{\prime \prime}$ & 5 & 3 & 959 & 30 & 110 & 215.9 & -26.5 & 226.8 & -15.3 & 7.6 & 102 & & \\
\hline 15 & 2 & $36^{\circ} 51^{\prime} 12.6^{\prime \prime}$ & $3^{\circ} 42^{\prime} 31.1^{\prime \prime}$ & 6 & 5 & 738 & 30 & 110 & 227.5 & -52.9 & 250.1 & -33.5 & 8.2 & 126 & & \\
\hline 16 & 2 & $36^{\circ} 51^{\prime} 12.6^{\prime \prime}$ & $3^{\circ} 42^{\prime} 31.1^{\prime \prime}$ & 6 & 5 & 719 & 30 & 110 & 225.7 & -25.3 & 234.2 & -10.1 & 4.4 & 236 & & \\
\hline 17 & 2 & $36^{\circ} 51^{\prime} 12.6^{\prime \prime}$ & $3^{\circ} 42^{\prime} 31.1^{\prime \prime}$ & 6 & 6 & 732 & 30 & 110 & 223.9 & -31.1 & 235.5 & -15.9 & 5.6 & 145 & & \\
\hline 18 & 2 & $36^{\circ} 51^{\prime} 10.8^{\prime \prime}$ & $3^{\circ} 42^{\prime} 39.5^{\prime \prime}$ & 6 & 4 & 351 & 30 & 110 & 219.8 & -26.0 & 229.7 & -13.2 & 16.2 & 330 & & \\
\hline 19 & 1 & $36^{\circ} 55^{\prime} 04.5^{\prime \prime}$ & $3^{\circ} 54^{\prime} 18.3^{\prime \prime}$ & 6 & 3 & 891 & 90 & 310 & 259.2 & -8.2 & 209.4 & -38.7 & 39.2 & 6 & 28.1 & 54.1 \\
\hline 20 & 2 & $36^{\circ} 51^{\prime} 54.0^{\prime \prime}$ & $3^{\circ} 43^{\prime} 36^{\prime \prime}$ & 8 & 6 & 694 & 40 & 110 & 210.4 & -27.2 & 225.5 & -14.3 & 3.1 & 326 & 50.0 & 6.8 \\
\hline 21 & 2 & $36^{\circ} 51^{\prime} 54.0^{\prime \prime}$ & $3^{\circ} 43^{\prime} 36^{\prime \prime}$ & 8 & 5 & 968 & 40 & 110 & 221.7 & -25.5 & 232.5 & -6.6 & 9.0 & 73 & & \\
\hline 22 & 2 & $36^{\circ} 51^{\prime} 57.0^{\prime \prime}$ & $3^{\circ} 43^{\prime} 30^{\prime \prime}$ & 7 & 6 & 1250 & 40 & 110 & 215.5 & -30.5 & 231.2 & -13.9 & 4.7 & 201 & & \\
\hline 23 & 2 & $36^{\circ} 51^{\prime} 51.0^{\prime \prime}$ & $3^{\circ} 43^{\prime} 32^{\prime \prime}$ & 8 & 5 & 1010 & 40 & 110 & 213.7 & -39.4 & 236.1 & -21.7 & 4.3 & 168 & & \\
\hline 24 & 1 & $36^{\circ} 30^{\prime} 23.5^{\prime \prime}$ & $2^{\circ} 14^{\prime} 06.5^{\prime \prime}$ & 8 & 7 & 550 & 50 & 160 & 284.9 & 48.1 & 219.4 & 50.5 & 5.1 & 141 & 218.1 & 8.0 \\
\hline 25 & 1 & $36^{\circ} 30^{\prime} 31.8^{\prime \prime}$ & $2^{\circ} 08^{\prime} 38.2^{\prime \prime}$ & 8 & 3 & 264 & 45 & 180 & 83.7 & -55.9 & 41.6 & -32.8 & 24.2 & 27 & 220.3 & 46.9 \\
\hline 26 & 1 & $36^{\circ} 30^{\prime} 32.7^{\prime \prime}$ & $2^{\circ} 08^{\prime} 40.5^{\prime \prime}$ & 7 & 4 & 180 & 45 & 180 & 60.4 & -49.2 & 36.7 & -17.9 & 30.2 & 10 & 215.4 & 50.3 \\
\hline 28 & 1 & $36^{\circ} 29^{\prime} 52.4^{\prime \prime}$ & $2^{\circ} 07^{\prime} 17.6^{\prime \prime}$ & 9 & 7 & 207 & 30 & 100 & 36.1 & 34.3 & 48.8 & 17.8 & 6.8 & 68 & 47.5 & 7.1 \\
\hline 29 & 1 & $36^{\circ} 28^{\prime} 38.0^{\prime \prime}$ & $2^{\circ} 13^{\prime} 13.1^{\prime \prime}$ & 5 & 3 & 0.06 & 38 & 65 & 84.4 & -13.8 & 94.2 & -48.7 & 37.9 & 12 & 272.9 & 68.5 \\
\hline 30 & & $36^{\circ} 29^{\prime} 00.5^{\prime \prime}$ & $2^{\circ} 14^{\prime} 11.6^{\prime \prime}$ & 8 & 4 & 10 & 35 & 140 & 3.3 & 26.0 & 25.2 & 47.2 & 20.3 & 21 & 23.9 & 30.7 \\
\hline 31 & 1 & $36^{\circ} 34^{\prime} 36.0^{\prime \prime}$ & $2^{\circ} 23^{\prime} 18.4^{\prime \prime}$ & 6 & 4 & 61 & 29 & 210 & 27.5 & 53.9 & 132.8 & 63.3 & 6.5 & 199 & 131.5 & 11.1 \\
\hline 32 & 1 & $36^{\circ} 34^{\prime} 38.7^{\prime \prime}$ & $2^{\circ} 23^{\prime} 15.8^{\prime \prime}$ & 6 & 6 & 86 & 29 & 210 & 41.2 & 51.5 & 124.1 & 55.8 & 3.6 & 355 & 122.8 & 5.8 \\
\hline 33 & 1 & $36^{\circ} 34^{\prime} 38.7^{\prime \prime}$ & $2^{\circ} 23^{\prime} 15.8^{\prime \prime}$ & 6 & 0 & 76 & 29 & 210 & - & - & - & - & - & - & - & - \\
\hline 35 & 2 & $36^{\circ} 27^{\prime} 43.9^{\prime \prime}$ & $2^{\circ} 37^{\prime} 45.7^{\prime \prime}$ & 14 & 12 & 24 & 58 & 16 & 301.5 & -67.8 & 222.4 & -35.2 & 4.4 & 98 & 41.1 & 5.4 \\
\hline 36 & 2 & $36^{\circ} 27^{\prime} 46.0^{\prime \prime}$ & $2^{\circ} 34^{\prime} 11.7^{\prime \prime}$ & 4 & 3 & 135 & 55 & 150 & 349.9 & 73.4 & 141.2 & 50.3 & 13.9 & 79 & 139.9 & 57.0 \\
\hline 37 & 2 & $36^{\circ} 26^{\prime} 20.1^{\prime \prime}$ & $2^{\circ} 26^{\prime} 16.1^{\prime \prime}$ & 6 & 3 & 191 & 50 & 10 & 18.3 & 68.5 & 13.2 & 18.7 & 3.9 & 28315 & 12.0 & 3.1 \\
\hline 38 & 2 & $36^{\circ} 26^{\prime} 44.1^{\prime \prime}$ & $2^{\circ} 26^{\prime} 28.9^{\prime \prime}$ & 6 & 6 & 684 & 27 & 1 & 79.3 & 79.8 & 20.9 & 59.4 & 4.3 & 242 & 19.6 & 8.5 \\
\hline 39 & 2 & $36^{\circ} 30^{\prime} 46.8^{\prime \prime}$ & $3^{\circ} 03^{\prime} 58.5^{\prime \prime}$ & 15 & 14 & 144 & 60 & 220 & 110.6 & 69.4 & 196.2 & 34.7 & 1.8 & 483 & 194.9 & 2.2 \\
\hline 40 & 2 & $36^{\circ} 30^{\prime} 46.8^{\prime \prime}$ & $3^{\circ} 03^{\prime} 58.5^{\prime \prime}$ & 6 & 6 & 890 & 60 & 220 & 112.2 & 75.1 & 202.9 & 33.5 & 4.6 & 216 & 201.6 & 5.5 \\
\hline 41 & 2 & $36^{\circ} 40^{\prime} 18.8^{\prime \prime}$ & $3^{\circ} 23^{\prime} 14.1^{\prime \prime}$ & 11 & 10 & 20 & 50 & 340 & 169.8 & -71.8 & 163.3 & -22.0 & 3.8 & 252 & 342.0 & 2.4 \\
\hline 42 & & $36^{\circ} 34^{\prime} 37.3^{\prime \prime}$ & $2^{\circ} 23^{\prime} 41.3^{\prime \prime}$ & 7 & 0 & 0.07 & 33 & 173 & - & - & - & - & - & - & - & \\
\hline 43 & & $36^{\circ} 34^{\prime} 21.5^{\prime \prime}$ & $2^{\circ} 23^{\prime} 07.5^{\prime \prime}$ & 6 & 0 & 1.0 & 36 & 152 & - & - & - & - & - & - & & \\
\hline 44 & 2 & $36^{\circ} 27^{\prime} 15.4^{\prime \prime}$ & $2^{\circ} 27^{\prime} 38.4^{\prime \prime}$ & 13 & 6 & 387 & 44 & 349 & 121.5 & 70.8 & 15.1 & 56.5 & 4.1 & 274 & 13.8 & 7.4 \\
\hline 45 & 2 & $36^{\circ} 27^{\prime} 07.4^{\prime \prime}$ & $2^{\circ} 26^{\prime} 36.2^{\prime \prime}$ & 10 & 4 & 221 & 21 & 318 & 317.3 & 0.8 & 317.3 & -20.2 & 4.9 & 346 & 136.0 & 5.2 \\
\hline 46 & 2 & $36^{\circ} 26^{\prime} 47.9^{\prime \prime}$ & $2^{\circ} 26^{\prime} 07.3^{\prime \prime}$ & 6 & 5 & 103 & 38 & 10 & 179.3 & -56.8 & 183.8 & -19.2 & 22.6 & 16 & 2.5 & 2.1 \\
\hline 47 & 2 & $36^{\circ} 28^{\prime} 17.9^{\prime \prime}$ & $2^{\circ} 30^{\prime} 47.5^{\prime \prime}$ & 15 & 14 & 72 & 22 & 10 & 100.7 & 54.8 & 72.5 & 49.5 & 3.2 & 156 & 71.2 & 4.9 \\
\hline 48 & 2 & $36^{\circ} 27^{\prime} 56.5^{\prime \prime}$ & $2^{\circ} 32^{\prime} 59.6^{\prime \prime}$ & 9 & 8 & 192 & 30 & 36 & 144.7 & 5.8 & 139.4 & 14.3 & 3.7 & 220 & 138.1 & 3.8 \\
\hline 49 & 2 & $36^{\circ} 27^{\prime} 49.7^{\prime \prime}$ & $2^{\circ} 34^{\prime} 10.2^{\prime \prime}$ & 9 & 9 & 93 & 55 & 150 & 321.8 & 72.6 & 154.0 & 52.1 & 4.2 & 152 & 152.7 & 6.8 \\
\hline 50 & 2 & $36^{\circ} 30^{\prime} 46.8^{\prime \prime}$ & $3^{\circ} 03^{\prime} 55.6^{\prime \prime}$ & 9 & 8 & 89 & 60 & 220 & 124.8 & 65.1 & 191.3 & 29.1 & 3.4 & 267 & 190.0 & 3.9 \\
\hline 51 & 2 & $36^{\circ} 28^{\prime} 12.3^{\prime \prime}$ & $2^{\circ} 31^{\prime} 19.8^{\prime \prime}$ & 8 & 6 & 40 & 26 & 25 & 185.8 & -56.1 & 192.6 & -31.2 & 10.9 & 41 & 11.3 & 10.2 \\
\hline
\end{tabular}

Note. Sites with coordinates, emplacement age (A) with 1 and 2 indicating 16-15 and 13-12 Ma (Ait-Hamou, 1987; Belanteur et al., 1995; Bellon et al., 1977; Hernandez \& Lepvrier, 1979), respectively, number of samples (n) and of samples allowing reliable data $\left(\mathrm{n}_{\text {ret }}\right), \mathrm{NRM}$ intensity (M) in $10^{-3} \mathrm{~A} / \mathrm{m}$, dip value (P) and direction $(\mathrm{G})$, declination $(\mathrm{D})$ and inclination $(\mathrm{I})$ before $\left(\mathrm{D}_{\mathrm{g}}, \mathrm{I}_{\mathrm{g}}\right)$ and after $\left(\mathrm{D}_{\mathrm{s}}, \mathrm{I}_{\mathrm{S}}\right)$ bedding correction, confidence angle $\left(\alpha_{95}\right)$, precision parameter $(\mathrm{k})$, rotation angle $(\theta)$ with its uncertainty $(\Delta \theta)$ according to Demarest (1983). Orange color for sedimentary sites; italics for site with scattered directions $(\mathrm{k}<40)$, bold for retained sites belonging to areas with large continuous outcrops. Blue frames correspond to quarries for which rotation has been determined by the mean of all their sites. 
(a)

(b)
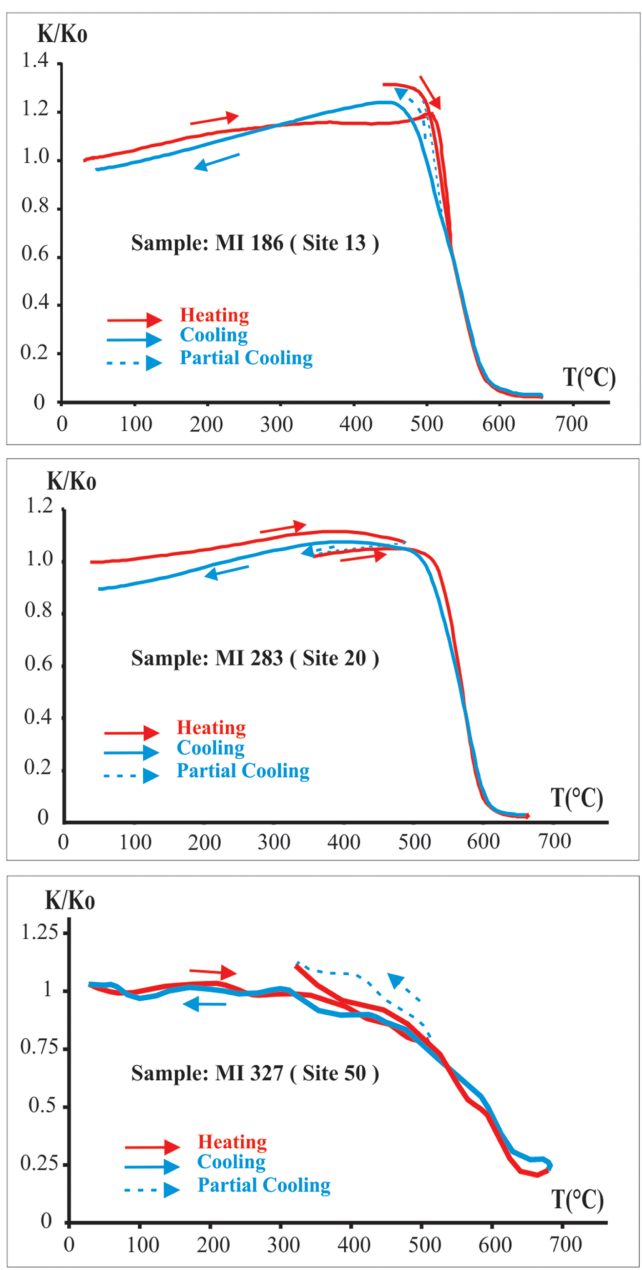

(c)
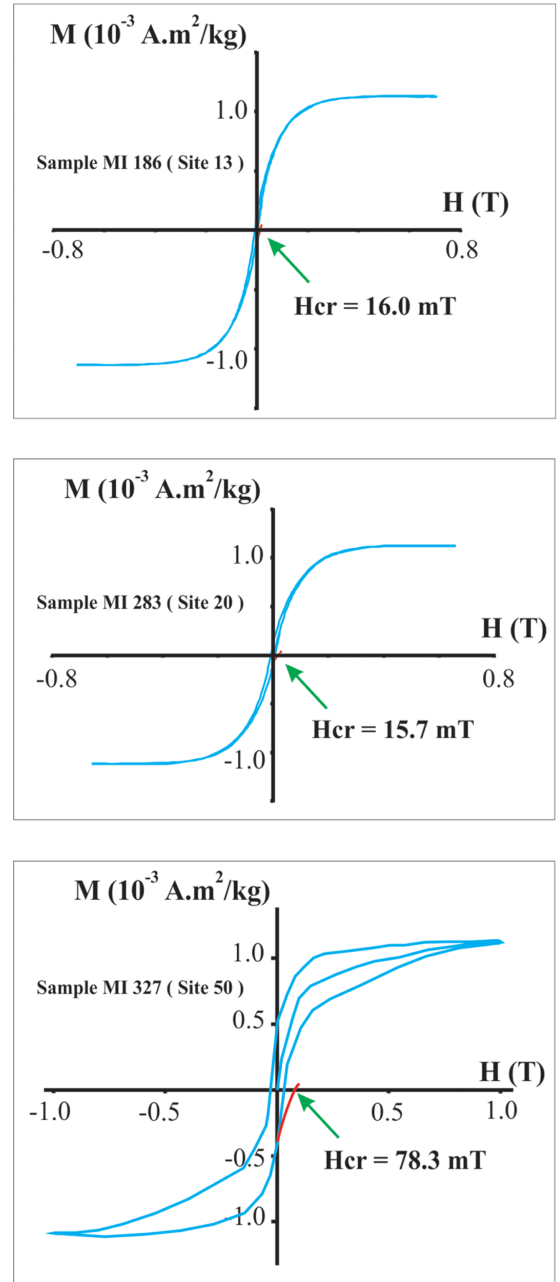

Figure 2. (a) Variation of the magnetization intensity during thermal demagnetization for representative samples, (b) typical thermomagnetic curves KT for Samples MI186, MI283, and MI327, and (c) typical hysteresis loops after correction for paramagnetism, for Samples MI186, MI283, and MI327. Hcr is the remanent coercive force, Field $\mathrm{H}$ in Tesla. 
Table 2

AMS Data From Thenia Granodiorite

\begin{tabular}{|c|c|c|c|c|c|c|c|c|c|c|}
\hline \multirow[b]{2}{*}{ Sites } & \multicolumn{2}{|c|}{ Coordinates } & \multirow[b]{2}{*}{$\mathrm{N}$} & \multirow{2}{*}{$\underset{\left(10^{-6} \mathrm{SI}\right)}{\mathrm{Km}}$} & \multicolumn{2}{|c|}{$\mathrm{K} 1$} & \multicolumn{2}{|c|}{$\mathrm{K} 3$} & \multirow[b]{2}{*}{$\mathrm{P}^{\prime}$} & \multirow[b]{2}{*}{$\mathrm{T}$} \\
\hline & $\lambda$ & $\phi$ & & & $\mathrm{D}\left({ }^{\circ}\right)$ & $\mathrm{I}\left({ }^{\circ}\right)$ & $\mathrm{D}\left(^{\circ}\right)$ & $\mathrm{I}\left({ }^{\circ}\right)$ & & \\
\hline 52 & $36^{\circ} 44^{\prime} 12.5^{\prime \prime}$ & $3^{\circ} 33^{\prime} 01.6^{\prime \prime}$ & 7 & 306 & 9.6 & 69.2 & 235.2 & 14.9 & 1.012 & 0.73 \\
\hline 53 & $36^{\circ} 44^{\prime} 11.7^{\prime \prime}$ & $3^{\circ} 33^{\prime} 07.9^{\prime \prime}$ & 5 & 249 & 336.2 & 28.1 & 243.7 & 4.6 & 1.015 & 0.24 \\
\hline 54 & $36^{\circ} 44^{\prime} 10.5^{\prime \prime}$ & $3^{\circ} 33^{\prime} 13.3^{\prime \prime}$ & 6 & 340 & 334.0 & 47.3 & 70.7 & 6.2 & 1.010 & 0.36 \\
\hline 55 & $36^{\circ} 44^{\prime} 15.2^{\prime \prime}$ & $3^{\circ} 33^{\prime} 15.6^{\prime \prime}$ & 6 & 260 & 339.2 & 11.2 & 245.3 & 19.1 & 1.014 & 0.31 \\
\hline 56 & $36^{\circ} 44^{\prime} 06.9^{\prime \prime}$ & $3^{\circ} 35^{\prime} 04.9^{\prime \prime}$ & 6 & 1323 & 298.9 & 83.2 & 63.9 & 3.9 & 1.028 & 0.22 \\
\hline 57 & $36^{\circ} 44^{\prime} 08.6^{\prime \prime}$ & $3^{\circ} 35^{\prime} 06.0^{\prime \prime}$ & 6 & 236 & 341.0 & 48.0 & 73.7 & 2.4 & 1.023 & 0.85 \\
\hline 58 & $36^{\circ} 44^{\prime} 09.3^{\prime \prime}$ & $3^{\circ} 35^{\prime} 09.8^{\prime \prime}$ & 6 & 265 & 348.3 & 4.9 & 258.2 & 0.8 & 1.010 & 0.03 \\
\hline 59 & $36^{\circ} 44^{\prime} 12.7^{\prime \prime}$ & $3^{\circ} 35^{\prime} 08.9^{\prime \prime}$ & 6 & 275 & 132.4 & 28.3 & 230.3 & 14.3 & 1.017 & 0.59 \\
\hline
\end{tabular}

Note. Sites with coordinates, number of samples (N), declination (D) and inclination (I) of maximum (K1) and minimum (K3) axes, and $\mathrm{P}^{\prime}$ and $\mathrm{T}$ parameters.

only weak mineralogical alteration occurred. For temperature higher than $500{ }^{\circ} \mathrm{C}$, the strong decrease of susceptibility, with a Curie point at about $550{ }^{\circ} \mathrm{C}$, indicates Ti-poor titanomagnetite as the principal mineral carrier. Most samples show an almost reversible curve during the total heating and cooling experiment, confirming that no major chemical alteration occurred. In a few sites, where unblocking temperatures are higher than $580{ }^{\circ} \mathrm{C}$, thermomagnetic curve decreases appear also for temperatures higher than $580{ }^{\circ} \mathrm{C}$, confirming the presence of hematite (Figure 2b, MI327 sample). In the Tipasa area (Figure 1), Aïfa (2014) found sulfides and hematite in the lava flows in addition to magnetite. Goethite also likely appears locally on some of his thermomagnetic curves.

Hysteresis loops show that the mean coercive force and the remanent coercive force, deduced from most samples, are low, about 7 and $19 \mathrm{mT}$, respectively (Figure 2c, MI186 and MI283 samples) and that the magnetization saturation is complete at approximately of 0.3 to $0.4 \mathrm{~T}$. This confirms the presence of a low coercivity component (like Tipoor titanomagnetite). However, few loops, related to samples having maximum unblocking higher than $580{ }^{\circ} \mathrm{C}$, show a wasp-waisted shape (Figure 2c, MI327 sample). They also have a higher partial saturation around $0.4 \mathrm{~T}$ and do not reach total saturation (indicating the presence of an additional high coercivity component). The mean coercive force and remanent coercive force are slightly higher in these cases (28 and $65 \mathrm{mT}$, respectively), confirming a mixing of low and high coercivity components (Ti-poor titanomagnetite and hematite).

In conclusion, Ti-poor titanomagnetite is the main magnetic carrier in these volcanic rocks, but, in some sites, hematite also was formed by oxidation of preexisting titanomagnetite.

\section{Magnetic Fabric of the Thenia Granodiorite}

As a complement to the paleomagnetic analyses, an Anisotropy of Magnetic Susceptibility (AMS) study was performed in an intrusive pluton located in a key position along one of the major faults structuring this area, to specify the displacements along this fault.

AMS is characterized by the principal susceptibilities $K_{1}, K_{2}$, and $K_{3}\left(K_{1} \geq K_{2} \geq K_{3}\right)$ and the mean susceptibility $\mathrm{K}_{\mathrm{m}}$. The Jelinek's (1981) parameters $\mathrm{P}^{\prime}$ and T were used to describe the magnetic fabric: $\mathrm{P}^{\prime}$ expresses the departure from a spherical AMS ellipsoid $\left(\mathrm{P}^{\prime}=1\right)$. The shape parameter $\mathrm{T}$ quantifies the shape of the magnetic ellipsoid, being linear when $-1 \leq \mathrm{T}<0$ and planar when $0 \leq \mathrm{T} \leq 1$. The orientation of the AMS is characterized by the magnetic foliation, equivalent to the $K_{1}-K_{2}$ plane ( $\perp$ to $K_{3}$ ) and the magnetic lineation, defined as direction parallel to $\mathrm{K}_{1}$. Data for a group of samples were analyzed using normalized tensor variability statistics (Hext, 1963; Jelinek, 1978).

The AMS is in fact a powerful tool to analyze emplacement conditions and deformation of intrusive bodies (e.g., Bouchez, 2000; Georgiev et al., 2014; Nouar et al., 2011; Tomezzoli et al., 2003). It could therefore highlight the structures within this Thenia pluton and their relationship with the Thenia fault. Forty-eight samples from eight sites were collected in two very large quarries (Figure 1 and Table 2). The magnetic fabric of this pluton is largely dominated by the paramagnetic minerals; only site 56 presents slightly higher susceptibility indicating a moderate ferrimagnetic contribution (Figure 3a). AMS data are characterized by dominant subhorizontal NNW-SSE magnetic lineations and vertical NNW-SSE magnetic foliations (Figure 3c). The fabric is always oblate (Figure 3b). 

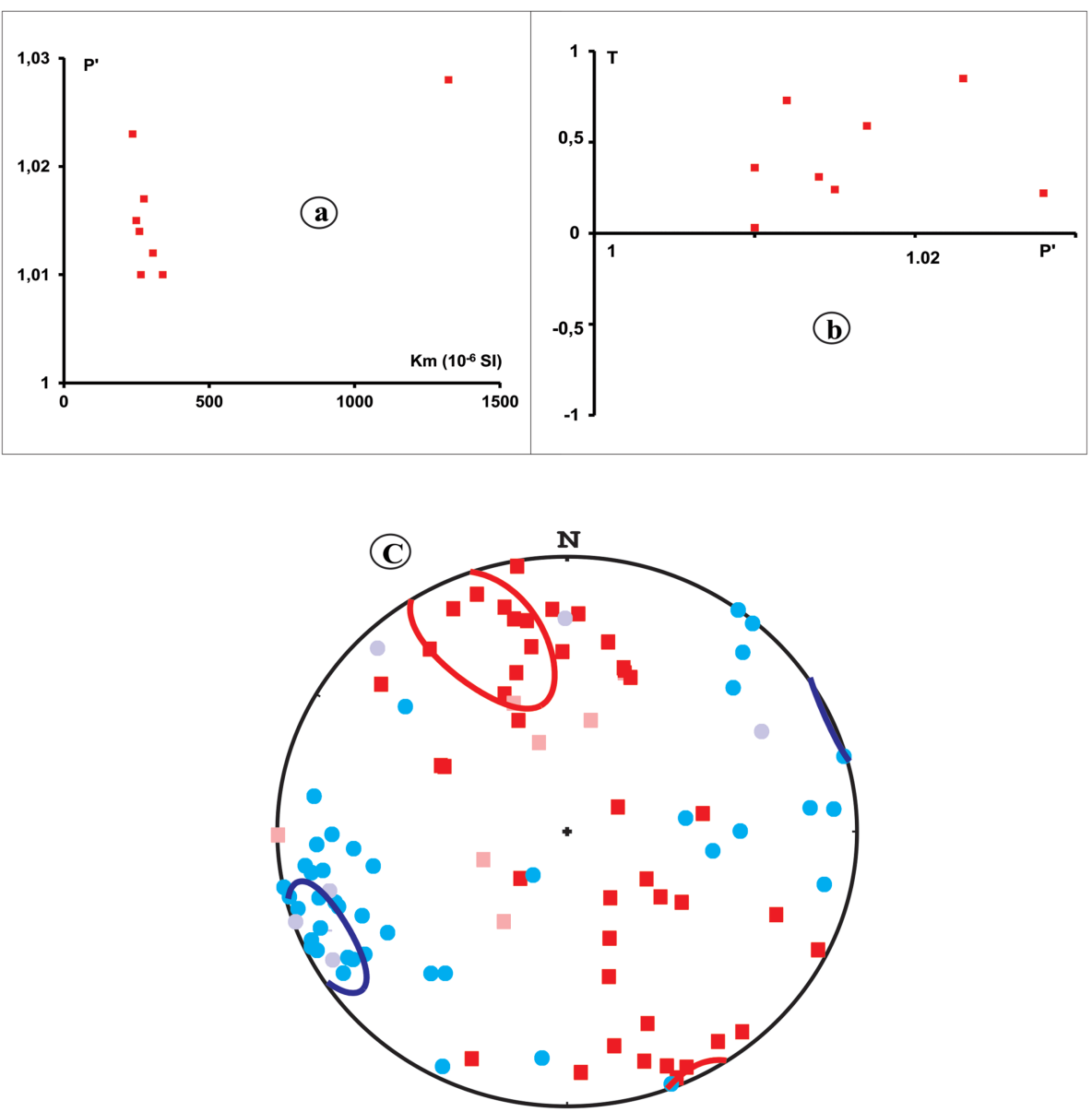

Figure 3. (a) P'Km and (b) P'T diagram (Jelinek, 1981) for the Thenia granodiorite; (c) maximum (squares) and minimum (circles) susceptibility axes of the Thenia pluton samples. Pink and violet colors correspond to the Site 56. Associated confidence zone was determined without the Site 56. Stereographic projection in the lower hemisphere.

\section{Paleomagnetic Results}

After selection from pilot specimen analysis, 328 samples have been demagnetized. For most of them, the AF procedure was applied; for the others, the TH treatment or a combined procedure (mixed AF-TH demagnetizations) was used. Within a site, no significant difference has been observed in the results obtained from the $\mathrm{TH}$ and AF demagnetizations. During the demagnetization process, two different main cases were observed from Zijderveld diagrams.

- The first one, obtained on most samples, is illustrated by a single stable B magnetic direction obtained after elimination of a secondary viscous A component. The Characteristic Remanent Magnetization B is of normal or reversed polarity according to the sites. Figure 4 shows examples of this behavior obtained from TH process (MI234 and MI274), AF method (MI142 and MI322), and mixed AF-TH one (MI319), respectively. This magnetic component is at least partially carried by the Ti-poor titanomagnetite. In fact, in some samples, hematite also appears to carry magnetization with the same orientation (Figure 4c).

- The other samples display erratic evolution, not allowing reliable paleomagnetic results. Four "volcanic" sites (1, 2, 7, and 33) gave scattered results. Three of these sites (1, 2, and 7) present weathered facies, and the other one (33) was probably affected by lightening. The sedimentary paleomagnetic data appear not to be usable in two sites (42 and 43), also because of unstable behavior during demagnetization. All these "unstable" sites are not considered further.

Finally, 43 sites (232 samples) allowed suitable paleomagnetic data. However, nine of them, presenting Fisher's k parameter lower than 40 (Table 1), can be only considered as rough indicator. Magnetization 


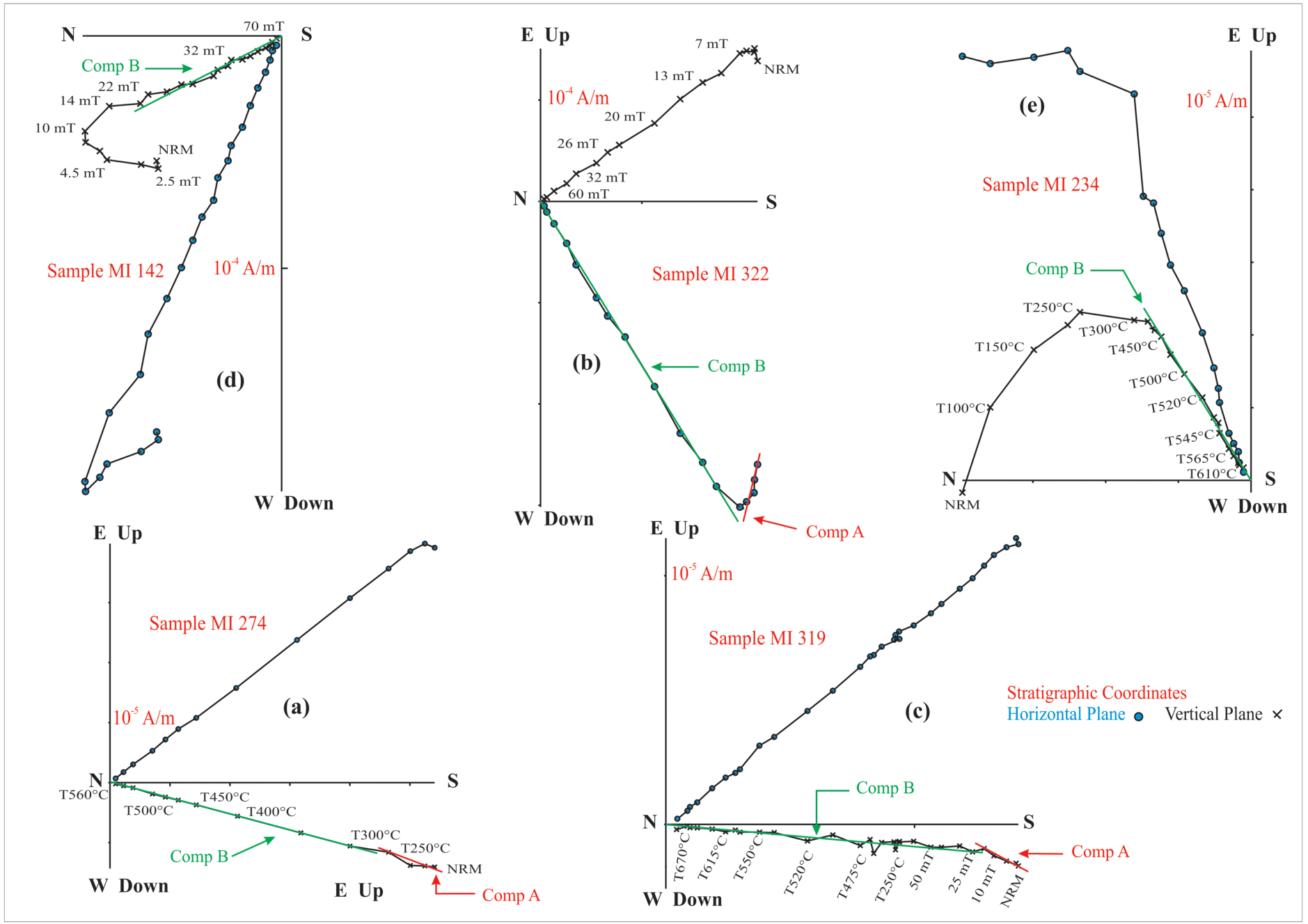

Figure 4. Orthogonal vector plots (filled circles = horizontal plane, crosses = vertical plane), in stratigraphic coordinates for Samples (a) MI274, (b) MI322, (c) MI319, (d) MI142, and (e) MI234.

polarity is normal for 15 sites and reversed for the 28 remaining sites. The mean inclination value after bedding correction is much lower for the reversed polarity $\left(-22.1^{\circ}\right.$; inclination between $-6.5^{\circ}$ and $-49.6^{\circ}$ as extreme values) than for the normal one $\left(42.2^{\circ}\right.$; inclination between $14.3^{\circ}$ and $\left.63.3^{\circ}\right)$. An "inclination only" fold test (Enkin \& Watson, 1996; Henry et al., 2010) has been performed considering the standard deviation of the distribution of inclination values during progressive unfolding (Figure 5a). The test result suggests a prefolding magnetization (minimum standard deviation between $50 \%$ and $110 \%$ unfolding) but should be considered cautiously because of the moderate variation of the standard deviation. In the few cases where they are outcropping, the sedimentary rocks under the lava flows have unfavorable characteristics for paleomagnetism, and no contact test has been obtained.

\section{Discussion}

\subsection{Magnetic Data}

7.1.1. Magnetic Fabric of the Thenia Pluton

The WNW-ESE-elongated Thenia pluton is limited to the south by the WNW-ESE Thenia major fault (Figure 1). This fault seems to be associated with the granodiorite intrusion. The Thenia granodiorite magnetic fabric has typical characteristics of a pluton emplaced under shearing conditions close to major faults, that is, vertical foliation, oblique to the fault plane and subhorizontal lineation (see Henry et al., 2019, and reference herein): For the WNW-ESE Thenia fault, vertical NNW-SSE-trending magnetic foliation direction 

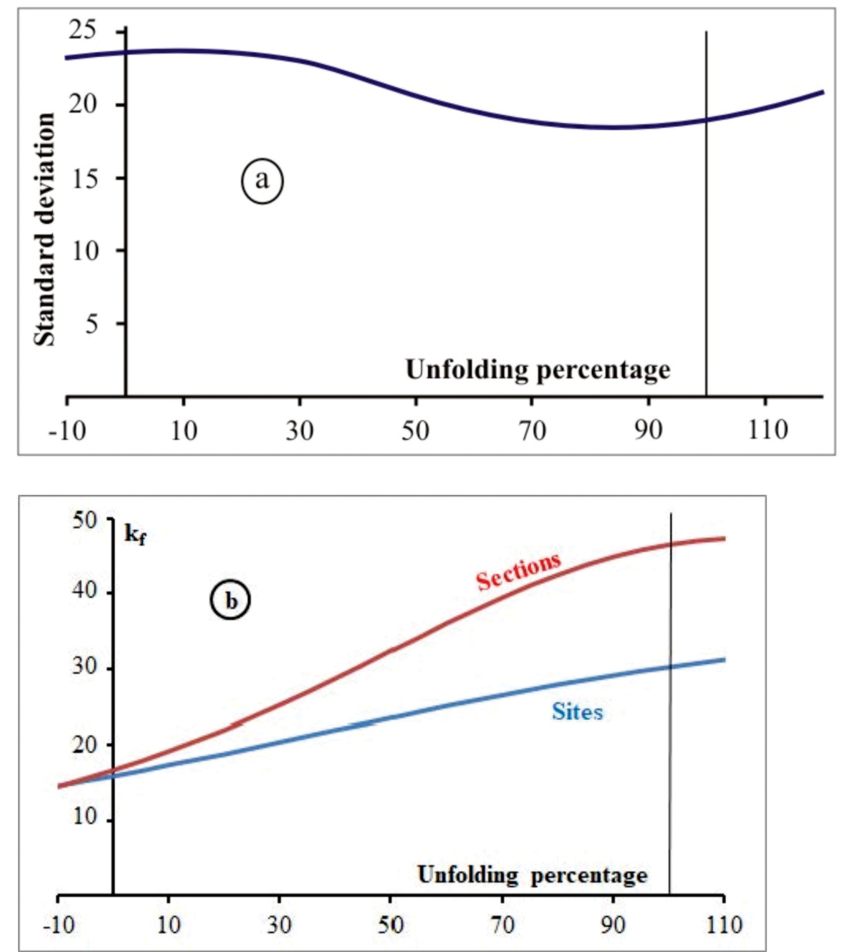

Figure 5. (a) Standard deviation of the distribution in inclination (in degrees) of the ChRM as a function of the untilting percentage for all the sites. (b) Fisher precision parameter $\mathrm{k}_{\mathrm{f}}$ as a function of the untilting percentage for the sites representative of the whole basin (17 individual sites or 5 sections; Sites 8 and 28 and Sections Merging Sites 3 and 4, 10-18, and 20-23).

clearly indicates a sinistral movement during the pluton emplacement, in contrast to the last known dextral movements (Figure 1)

\subsubsection{Paleomagnetic Data}

\subsubsection{Magnetic Properties}

The Sites 3 to 7 along the coastline are strongly weathered. The amount of remaining magnetite is variable and often very weak, the magnetite being mostly altered into goethite. The hematite is present in some sites on the basin borders within fault zones, although weathering seems to be negligible in some outcrops. The situation could be related to the effects of fluids in these sites within such a strongly fractured context. The obtained paleomagnetic directions for temperatures steps lower and higher than $580{ }^{\circ} \mathrm{C}$ are almost similar for different samples, suggesting that a partial oxidation occurred shortly after the lava emplacement. This similarity could be also explained by a composite character of the remanent magnetization for steps below $580{ }^{\circ} \mathrm{C}$ (magnetization related to both magnetite and hematite). Nevertheless, main rotations (see below) occurred after the acquisition of the remanent magnetization.

\subsubsection{Structural Corrections}

As shown in many studies of volcanic rocks, the paleomagnetic directions in different lava flows present relatively important scattering, mainly due to the secular variation. Moreover, in such studies, the bedding correction could be disputable. In the present study and for sites not belonging to large blocks, the relation with surrounding rocks is not clear, and the structural corrections have large uncertainty. At least in one site (Neck close to Cap Djinet, Site 9), the sampled rocks belong to intrusions. In cases of sill, the observed dip may have been acquired before the intrusion (e.g., Smith et al., 2007). Structural corrections could complicate matters, since a slope may already have existed at the emplacement time (Silva et al., 2018). AMS data obtained on 18 samples from Sites 20-23 indicated lava likely flowing toward ESE (ENE before block rotation), suggesting a possible weak dip with this orientation (corresponding to the present dip) during the lava emplacement (e.g., Cañón-Tapia et al., 1995; Plénier et al., 2005; Thompson et al., 1986). 

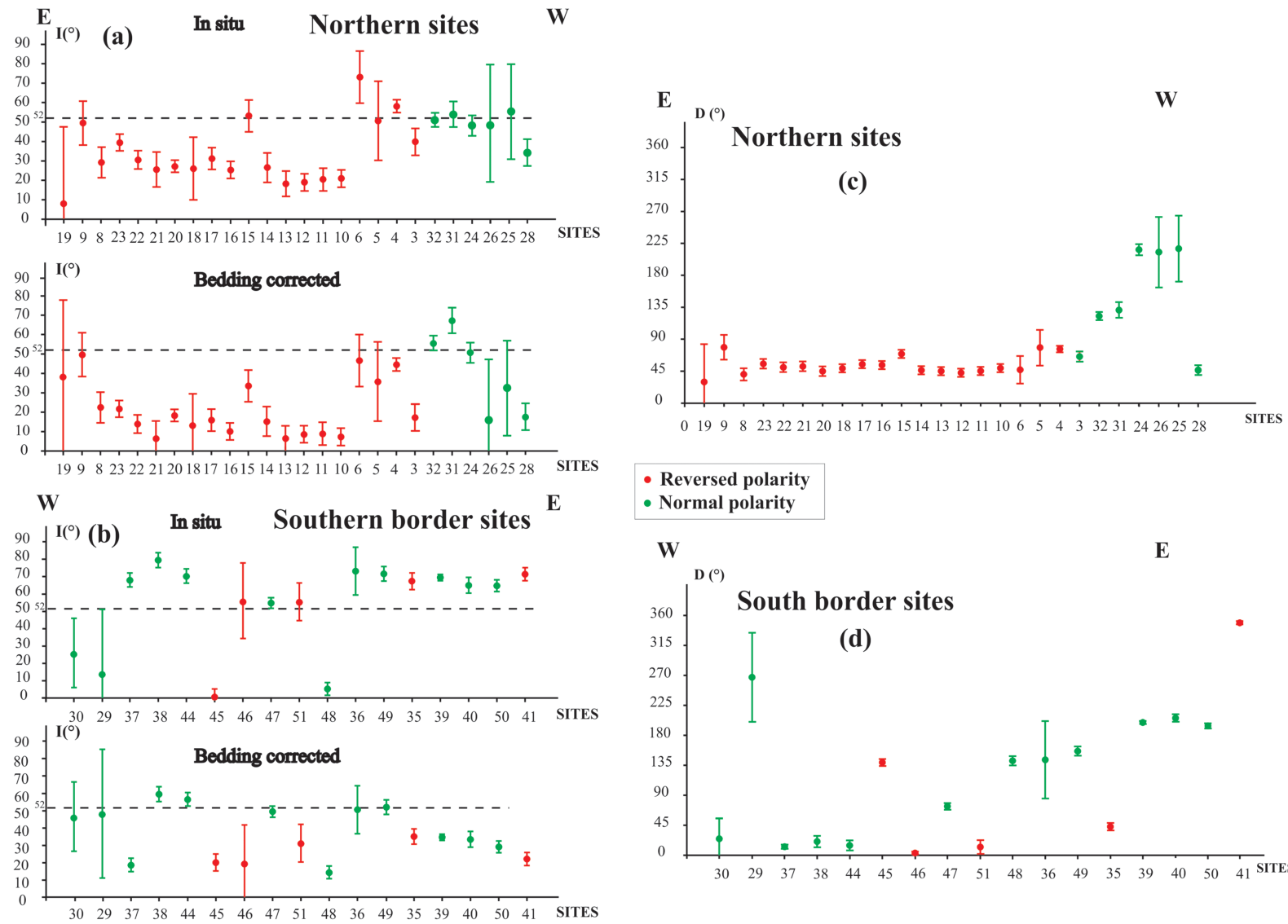

- Reversed polarity

- Normal polarity

W

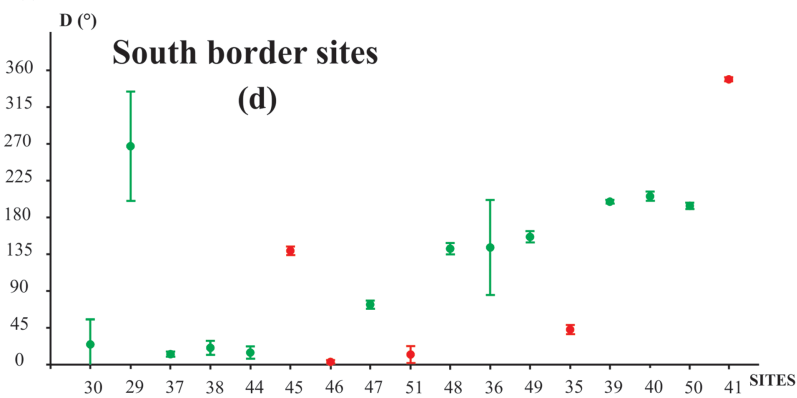

Figure 6. (a and b) Magnetic inclination (recalculated as positive value) with $\alpha_{95}$ uncertainty according to the sites: sites with normal (green) or reversed (red) polarity; the horizontal line represents the expected inclination value $\left(52^{\circ}\right)$; (c and d) magnetic declination (recalculated with normal polarity) with its $\Delta \theta$ uncertainty (Table 1) according to the sites: sites with normal (green) or reversed (red) polarity. Order of the sites along the diagrams corresponds to longitude.

\subsubsection{Paleomagnetic Directions}

No clear difference can be evidenced for the paleomagnetic direction according to the emplacement age (Table 1). The obtained magnetic inclinations derived from all data are shown (all presented with normal polarity for a clear comparison) on Figures $6 \mathrm{a}$ and $6 \mathrm{~b}$ (cases with initial reversed polarities are indicated by red color in figures). This shows that, in the northern margin of the basin, the reversed polarity has been obtained in all sites but six sites (all from the northwestern border), while the polarity is predominantly normal on the southern margin sites ( 12 normal for 5 reversed). Both polarities have also been obtained for the sites of the oldest as well as the youngest groups of age.

The mean inclination values $\left(42.2^{\circ}\right.$ and $-22.1^{\circ}$ for the normal and reversed polarities, respectively) show that part of the sites have unusually low magnetic inclination compared to that expected for Miocene age (Figures 6a and 6b), based on the Apparent Polar Wander Path of Besse and Courtillot (2002). The low inclination values determined in volcanic rocks cannot be due to an inclination shallowing like in sedimentary rocks (e.g., Krijgsman \& Tauxe, 2004). AMS is very weak (see Hrouda \& Ježek, 2017), of the order of 4\%, and principal susceptibility axes are relatively scattered (test made on 18 samples from Sites 20-23) and cannot account for the low inclination values (e.g., Gattacceca \& Rochette, 2002). There are 13 sites with low inclinations, all corresponding to the two neighboring quarries with lava piles (Figure 7a), while some other sites also present low inclination, even on the southern border. A strong local tilting, of about $30^{\circ}$, of large block (including at least the two quarries), before lava emplacement, should have been observed in the overlying sedimentary deposits. So this assumption cannot be considered as reliable. The low inclination is then likely related to the magnetic field behavior (e.g., Di Chiara et al., 2012). In our case, the presence of sites with higher inclination values indicates that the low inclination was related to a temporary phenomenon, likely corresponding to a reversal or an excursion of the Earth magnetic field. 

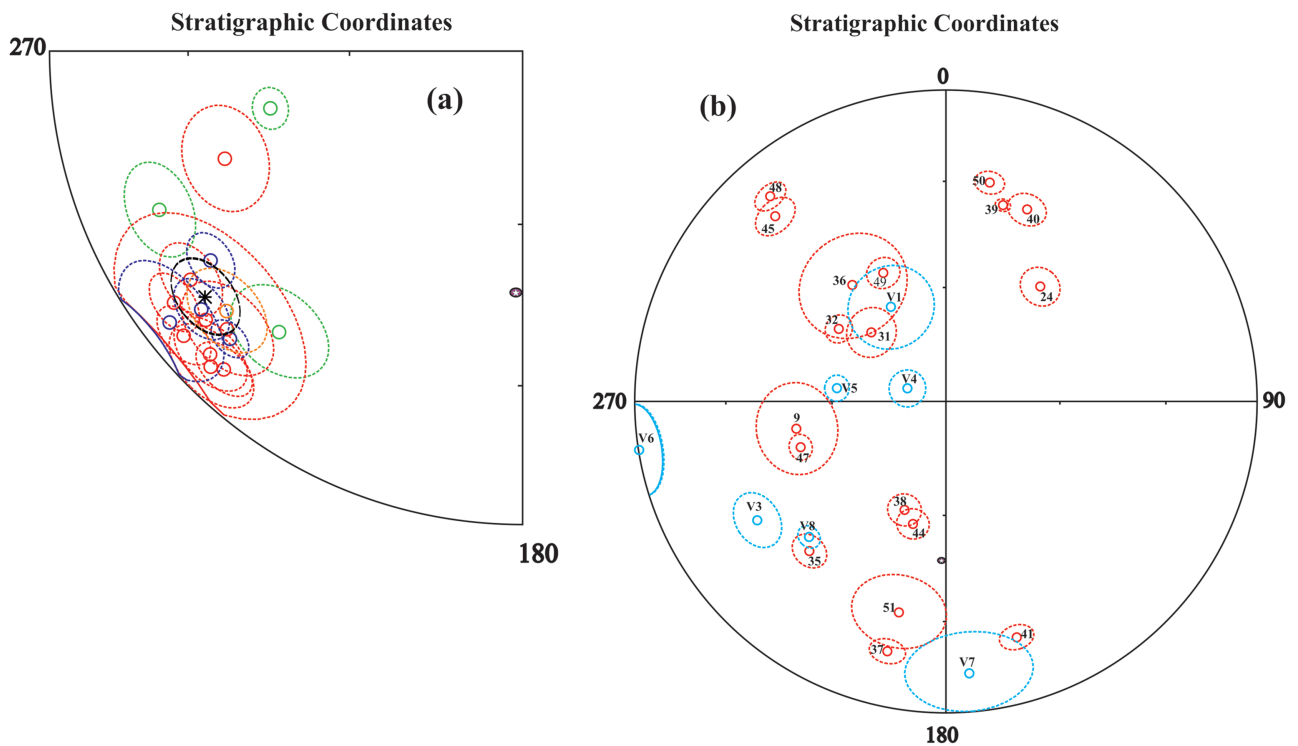

Figure 7. Equal-area plot after dip correction of the mean-site paleomagnetic directions with associated $95 \%$ confidence zone in lava flows: (a) sites reliable for basin-scale data: 3-4 and 8 (green), 10-18 (red), 20-23 (blue), and 28 converted to negative inclinations (orange). The mean direction with its confidence zone is in black. (b) Sites affected by local rotations, all converted to negative inclinations: this study (red) and from Aiffa, 2014 (blue). Continuous (dotted) line for confidence circles correspond to lower (upper) hemisphere.

Figures $6 \mathrm{c}$ and $6 \mathrm{~d}$ display a synthetic presentation of the magnetic declinations (after dip correction), with associated uncertainty (Table 1) obtained from the different sites (declinations corresponding to reversed polarity were recalculated as having a normal polarity). The scattering of the declinations clearly evidences that different rotations occurred according to the location within the basin. Expected declination for the lava age in the Mitidja basin being $D_{\text {ref }}=1.3^{\circ}$ (Besse \& Courtillot, 2002), a rotation $\theta$ since the lava emplacement for each site is evaluated from the difference between the measured declination and $\mathrm{D}_{\text {ref. }}$. In Table 1 , the uncertainty $\Delta \theta$ on $\theta$ has been determined using the Demarest (1983) approach that gives similar results as using the Deenen et al. (2011) one.

The northeastern sites (3-7, 10-18, and 20-23; Figure 1) correspond to areas with very large continuous outcrops, that is belonging to large blocks and being associated with reliable structural corrections. (Data for Sites 5 and 6, associated with a very large uncertainty $-\mathrm{k}<40-$ Table 1 , have been not considered.) A similar paleomagnetic direction, but with opposite polarity, has been found in Site 28, taken from a thick lava flow and belonging to a large (more than $1 \mathrm{~km}$ ) block with coherent dip, limited to the north and to the south by two E-W important faults and corresponding to the western closure of the basin (Figure 1). For all these large blocks, the mean direction $\left(\mathrm{N}=17\right.$ sites, $\mathrm{D}=224.2^{\circ}, \mathrm{I}=-31.8^{\circ}, \mathrm{k}=20$, and $\alpha_{95}=8.2^{\circ}$ and $\mathrm{D}=232.3^{\circ}, \mathrm{I}$ $=-16.5^{\circ}, \mathrm{k}=37$, and $\alpha_{95}=5.9^{\circ}$, before and after dip corrections, respectively) can be therefore considered as representative for the "stable" parts of the Mitidja basin (the mean direction, calculated only for the eastern data, i.e., without the site 28 , is not significantly different: $\mathrm{N}=16, \mathrm{D}=224.7^{\circ}, \mathrm{I}=-31.6^{\circ}, \mathrm{k}=19$, and $\alpha_{95}=$ $8.7^{\circ}$ and $\mathrm{D}=232.5^{\circ}, \mathrm{I}=-16.4^{\circ}, \mathrm{k}=35$, and $\alpha_{95}=6.3^{\circ}$ before and after dip corrections, respectively). A positive fold test (see Figure $5 \mathrm{~b}$ ) attests of the reliability of these paleomagnetic data. To avoid an overly dominant effect of the quarries data, a fold test was also performed by sections (merging sites from same areas), and it is also clearly positive (Figure $5 \mathrm{~b}$ ). These reliable paleomagnetic data indicate that the basin underwent an average clockwise rotation of $48 \pm 17^{\circ}$. The fact that most northeastern sites correspond to paleomagnetic directions acquired during a period with unusual direction (relatively low inclination) of the magnetic field (reversal or excursion) could have introduced an incorrect interpretation of these directions as rotation indicators for the whole basin. However, similar rotation angles have been obtained in neighboring sites (4, 6, and 9) associated with paleomagnetic inclination expected for Miocene age, supporting thus the reliability of this interpretation. 


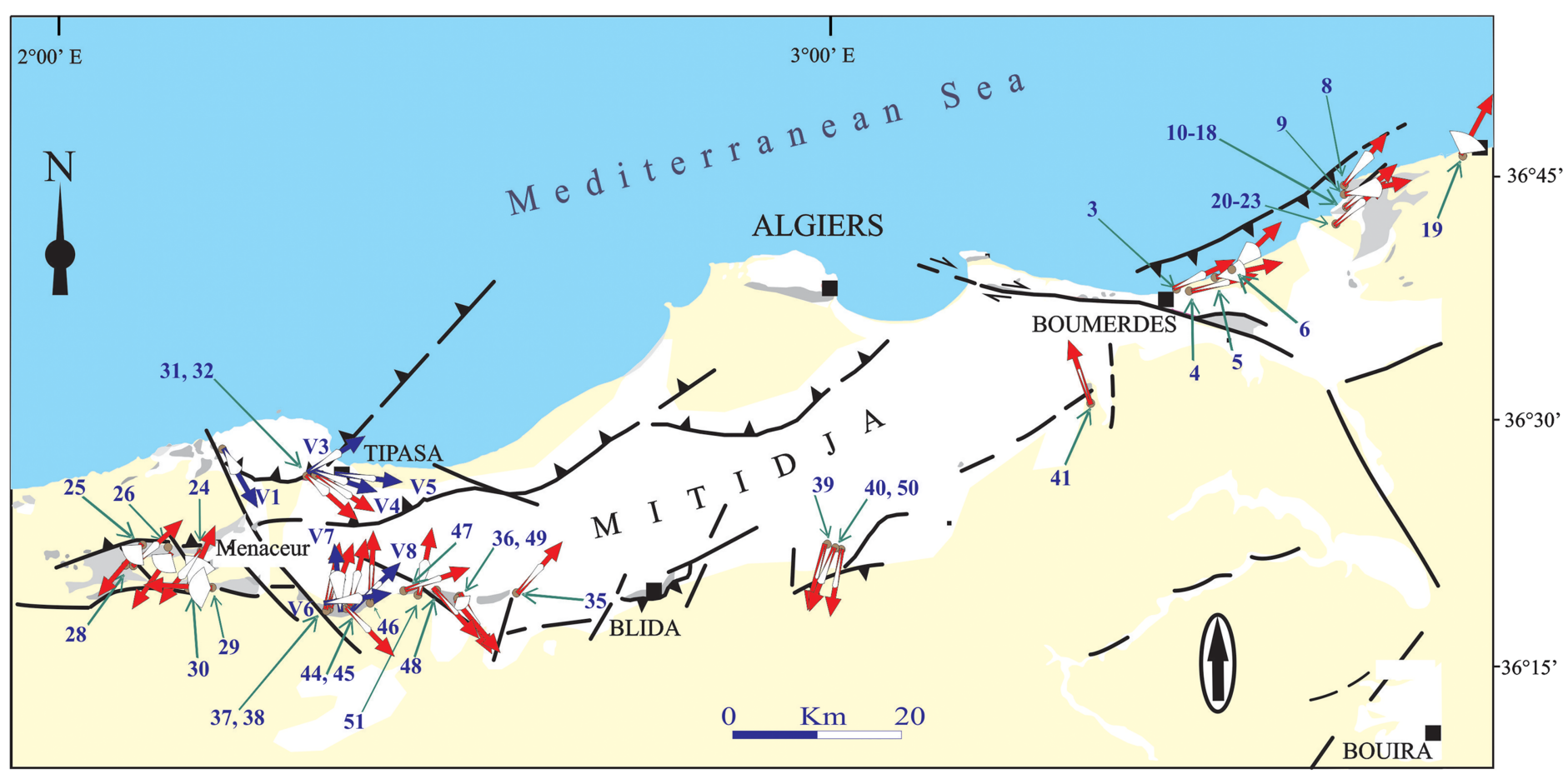

Figure 8. Map with arrows indicating the paleomagnetic declinations (all presented as normal polarity), with, in white, their associated confidence cones (Table 1), obtained in the different sites: blue arrows for sites with magnetite as magnetization carrier in Aïfa (2014) and red arrows for this study. The black arrow on the bottom right indicates the expected paleomagnetic direction after Besse and Courtillot (2002).

The other studied sites are all (except one: Site 19) located on the southern and western basin borders along faults (Figure 1) and correspond only to small outcrops, without any continuity. They likely belong to small blocks, embedded within the fault zones. Moreover, for some of them, structural corrections to apply are not always clear. As indicated before, the only aim of the study of these sites was determination of local tectonic movements, keeping in mind that in few cases, additional uncertainty could be related to structural corrections. The obtained paleomagnetic declinations indicate that, since the lava emplacement, rotations affected these sites (average rotation magnitude: $98^{\circ}$ ). The associated very large uncertainty for this mean rotation $\left( \pm 72^{\circ}\right)$ clearly shows that these sites underwent different various local rotations, often of large amplitude, and that a mean paleomagnetic direction and a mean rotation should be meaningless. The result of these inhomogeneous rotations is a misleading random distribution of the paleomagnetic directions (Figure 7b). It is then obvious that a classical fold test is not applicable for such data. For a rough estimate of the structural evolution within and close to the faults, it is interesting to compare (Figure 6) the rotation undergone by these sites with that of the global rotation of the basin (which is in average about $48^{\circ}$ clockwise), that is, to determine the local "additional" rotation for these sites. Two cases for such additional rotation can be clearly distinguished on the Figure 6 .

- Some sites should have been affected only by moderate counterclockwise movement relatively to the stable Africa. Most of them are located on the southwestern border of the basin (Sites 37, 38, and 44), in the same restricted area (Figure 8) that could have been a fault-bounded slice less rotated than the main part of the basin.

- For all other sites (belonging mostly to the southern border), this additional rotation was much stronger and clockwise. The context of dextral shearing, giving global clockwise rotation at the basin scale, also appears at a more local scale, along faults giving additional clockwise rotation.

On the northern side of the Mitidja basin, around the Chenoua massif (Tipasa area), paleomagnetic directions (Aïfa, 2014; this study), mostly obtained within fault zones, show clockwise rotations relatively to stable Africa (Figure 8).

\subsection{Geodynamical interpretations}

\subsubsection{Structural Implications}

The onshore Mitidja basin is longer than $150 \mathrm{~km}$, and its offshore eastern limit is not well identified. A global rotation of the entire basin as a single block is not a realistic assumption because of the lack of other evidence 
over the Northwestern African margin. Therefore, considering the homogeneous rotations in the "stable" parts of the Mitidja basin, the rotation angle indicates a coherent movement of several independent large blocks. The obtained data in this study are discussed in the context of the bookshelf tectonics model (see Mandl, 1987), proposed by Meghraoui et al. (1996) for the Tell Atlas. Such a model is supported by the presence of the Sahel anticline (fault-related fold) on the northern basin margin and of NE-SW and NW-SE trending tectonic structures. We mention that bookshelf tectonics was already proposed for the Cheliff basin located west of the Mitidja basin (Aïfa et al., 1992; Derder et al., 2011).

For the Mitidja basin, clockwise rotations of different blocks should lead to the existence of sinistral faults, presently roughly NE-SW, at block limits. Quaternary deposits in the inner part of the basin does not allow direct observation of such faults, but the southern margin of the basin is compartmented by several NNESSW trending sinistral faults (Figure 1) (Maouche et al., 2011; Maouche \& Harbi, 2018)

The paleomagnetic data show that the western and southern Mitidja basin borders are characterized by multiple strong rotations (Figure 8). This variability argues for the existence of several small blocks implying a different rotation rate for one block with respect to another. Similar deformation pattern has been reported at "Beni Haoua" area, west of the Mitidja basin on the coastal area (Derder et al., 2013). As mentioned above, the southern Mitidja border is constituted of small blocks, interpreted as accommodating the rotation, (at the contact with nonrotated area located southward), through local fragmentation of the large rotated blocks into small independent blocks. These rotations are comparable to those deduced in the "Beni Haoua" zone. As a result, the southern Mitidja border can also be interpreted as corresponding to a narrow highly deformed roughly E-W trending major shear zone.

Another interesting result is that the Thenia fault, presently dextral, was sinistral during Thenia pluton emplacement (15 Ma; Belanteur et al., 1995); it could reflect an effect of the clockwise block rotation, at the limit of the rotated blocks, before the dextral regional shearing along this fault.

It is well known that the bookshelf tectonics theoretical model stipulates the opening of a free "space zone" at the ends of the rotated blocks (Wernicke \& Burchfiel, 1982). These "open spaces" are filled by smaller blocks resulting from the fragmentation at the ends of the main rotated blocks. Such tectonic evolution could correspond to an early period of the Mitidja main block rotation (Algiers block in Meghraoui \& Pondrelli, 2012). Afterward, reverse faults and folds growth can therefore be expected on the southern and northern borders. This is due to the fact that the global N-S shortening, generating $2.0 \mathrm{~mm} / \mathrm{yr}$ uplift on the segmented Sahel fold (Maouche et al., 2011), is also associated with sinistral strike-slip movements along the NE-SW trending faults.

Fold test (Figure 5) argues for primary magnetizations, indicating that these rotations occurred after the emplacement of the considered volcanic rocks.

7.2.2. Accommodation of the Africa-Eurasia Convergence in the Mitidja Basin

The tectonic related to Africa-Eurasia convergence since the Late Cenozoic is partly accommodated by shortening in the Tell Atlas fold-and-thrust belts, whereas faults system sets separated crustal blocks. This fault system associated with NE-SW trending "en echelon" sinistral thrust defines several NE-SW-oriented tectonics blocks. This deformation pattern is consistent with the distribution of seismicity (see Ousadou et al., 2014) and geodetic studies (Serpelloni et al., 2007). Geological investigations show that this tectonic activity is absorbed by the 2 to $3 \mathrm{~mm} / \mathrm{yr}$ rate shortening in the Tell Atlas (Meghraoui \& Doumaz, 1996).

Results about Thenia pluton (AMS) and Blida faults system (paleomagnetism) underline the importance, in the structural evolution of the Mitidja basin, of the E-W to WNW-ESE trending main regional structures that likely separate areas with different rotation magnitudes. The initial sinistral movement along the Thenia fault, evidenced by the magnetic fabric of the Thenia pluton and the fragmentation in "small" blocks on the southern border of the basin would represent an effect of the beginning of bookshelf evolution (accommodation of the block rotation at the limits of the system). The block rotation ended when it reached the presently observed angle. This angle could correspond to a maximum possible N-S shortening of the basin without disruption and total fragmentation of the largest rotated blocks. The subsequent accommodation stage would have been transferred to dextral movement along the major E-W to WNW-ESE trending structures. Paleomagnetic data already evidenced block rotation in the Cheliff basin (Aïfa et al., 1992; Derder et al., 2011, 2013) and in Oran volcanic provinces (Derder et al., 2018), confirming that all the Tell Atlas area was affected by active faulting and transpression tectonics (Meghraoui \& Pondrelli, 2012). Figure 9 shows EW to WNW-ESE trending faults as major structures separating areas where blocks, affected by vertical axis 


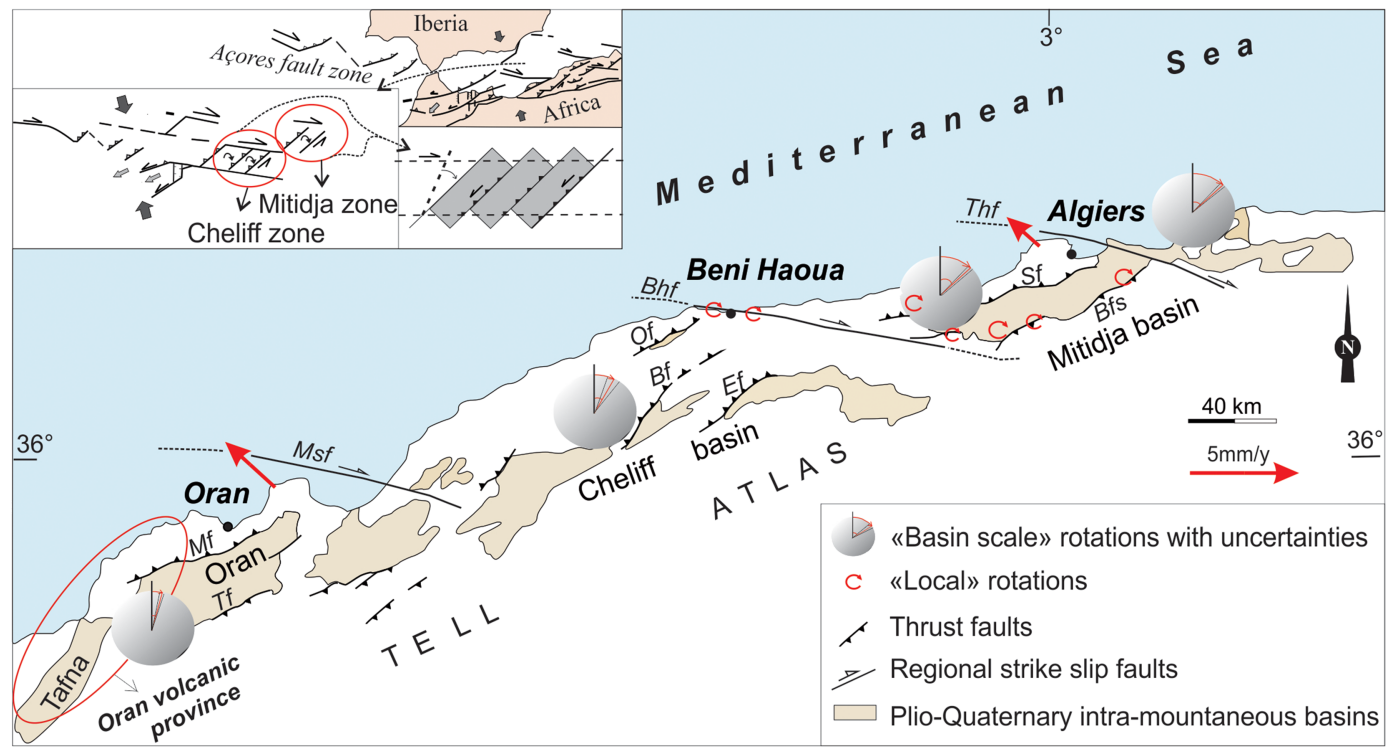

Figure 9. Synthetic map showing the block rotations in the Central and Western Tell of Algeria according to paleomagnetic data. Red arrows show the velocity field in a Eurasia fixed reference frame (Nocquet, 2012). Basin-scale block rotations in Oran volcanic provinces; Cheliff and Mitidja basins correspond to the mean reliable (i.e., not in local shear zones context) paleomagnetic data (Derder et al., 2011, 2013, 2018). "Local" rotations on the contrary are associated with data from these sites embedded within fault zones. Thf $=$ Thenia fault; $\mathrm{Sf}=$ Sahel fault; $\mathrm{Bfs}=$ Blida faults system; $\mathrm{Bhf}=$ Beni Haoua Strike slip; Of $=$ Oued Allalah thrust Fault; $\mathrm{Bf}=$ Boukadir thrust fault; $\mathrm{Ef}=\mathrm{El}$ Asnam thrust Fault; $\mathrm{Msf}=$ Mostaganem Fault; $\mathrm{Mf}=$ Murdjadjou thrust Fault; $\mathrm{Tf}=$ Tamazoura thrust fault $(\mathrm{faults}$ location are from Meghraoui, 1988, and Meghraoui et al., 2004). The inset illustrates the example of bookshelf block rotation model of the Cheliff-Mitidja areas (Meghraoui et al., 1996, Derder et al., 2011; Meghraoui \& Pondrelli, 2012, this paper).

rotation, are bounded by sets of parallel faults, presently NE-SW trending and mostly of hidden nature. These faults accommodated the relative motion between blocks in the regional N-S shortening process. Rotation amplitude within the studied basins increases from Oran volcanic provinces to Cheliff and to Mitidja, that is, from West to East and, considering the compartments separated by the major E-W structures, from South to North (Figure 9).

\section{Conclusion}

It has been established that the bookshelf tectonic model can account for the Cheliff basin tectonics, as part of the process of accommodating the Africa-Eurasia convergence. In the present study, in the Mitidja basin, new paleomagnetic data demonstrate coherent clockwise rotations of large blocks. They show that the bookshelf mechanism also affected the Mitidja area, in a context of reactivation of E-W trending structure (Meghraoui et al., 1996). Both the direction of plate convergence and the observed structures within the basins seem to be the main factors that control the rate and the migration of the block rotations within the Northwest Africa margin. This suggests that, when a blocking limit of the Mitidja system is reached, the increase in block rotations should be probably more intense westward in the Cheliff and Oran volcanic provinces areas in order to accommodate the ongoing collision.

For the Tell Atlas, a simple model is derived from Morel and Meghraoui et al. (1996) and Meghraoui et al. (1996). E-W to WNW-ESE trending major structures separate independent bookshelf system areas. With this model, the "Beni Haoua" and the southern border Mitidja shear zones should be narrow zones of major deformation. The Beni Haoua major structure separates the Cheliff and Mitidja basins (Figure 9). The initial sinistral movement along the Thenia fault indicates that this last structure could represent the northern border of some rotated blocks evidenced in the Mitidja basin. Rotations north of the Thenia fault, in the Cap Djinet area, should correspond to another bookshelf system located further north.

The remaining question is whether a similar evolution may occur along the North African margin toward the West and East of the Mitidja-Cheliff-Northwestern Algeria areas, that is, in Morocco and Eastern Algeria-Tunisia. Further East, paleomagnetic investigation of the western Sicily Maghrebian belt has 


\section{Acknowledgments}

This project is supported by the Algerian-French collaboration CMEP program $07 \mathrm{MDU} 712$. We wish to express our thanks to Karim Meziane and Christopher Day for their help with the writing the manuscript, to Mustapha Meghraoui for useful discussions, and to Cor Langereis, an anonymous reviewer, and the Associate Editor for detailed and helpful comments. We are also very grateful to the civil and military authorities for their constant help in the field. The data are available from the following link: ftp.craag.dz (using "anonymous" as username) revealed that large clockwise rotations with respect to the Hyblean-African foreland have occurred since the Tertiary (Channell et al., 1990; Schult, 1976; Speranza et al., 2018).

\section{References}

Aiffa, T. (2014). Neogene rotations of the Chenoua massif, northern Algeria, from remagnetizations. Arabian Journal of Geosciences, 7(11), 4629-4640. https://doi.org/10.1007/s12517-013-1124-x

Aïfa, T., Feinberg, H., Derder, M.E.M., \& Merabet, N. (1992), Rotations paléomagnétiques récentes dans le bassin du Chéliff (Algérie). Compt. rend. Acad. Sci., Paris. 314, II, 915-922.

Ait-Hamou F. (1987), Etude pétrologique et géochimique du volcanisme d'âge miocène de la région de Hadjout (Ouest Algérois). Magister thesis, U.S.T.H.B. University Algiers, 269p.

As, J. A., \& Zijderveld, J. D. A. (1958). Magnetic cleaning of rocks in paleomagnetic research. Geophysical Journal of the Royal Astronomical Society, 1, 308-319.

Ayadi, A., S. Maouche, A. Harbi, M. Meghraoui, H. Beldjoudi, F. Ousadou, et al. (2003), Strong Algerian earthquake strikes near capital city. EOS Trans., Amer. Geophys. Union 84, 561.

Belanteur, O. (2001), Le magmatisme miocène de l'Algérois: Chronologie de mise en place, pétrologie et implications géodynamiques. PhD thesis, Université de la Science et de la Technologie Houari Boumediène, Algiers, Algeria $232 \mathrm{p}$.

Belanteur, O., Bellon, H., Maury, R.C., Ouabadi, A., Coutelle, A., Semroud, B., et al. (1995), Le magmatisme miocène de l'Est Algérois: Géologie, géochimie et géochronologie ${ }^{40} \mathrm{~K}-{ }^{40} \mathrm{Ar}$, Compt. rend. Acad. Sci. Paris, IIa 321, 489-496.

Bellon H., \& Brousse, R. (1977), Le magmatisme périméditerranéen occidental. Essai de synthèse, Bull. Soc. géol. France, XIX, (7), 469-480.

Bellon, H., Lepvrier, C., Magné, J., \& Raymond, D. (1977). L'activité éruptive dans l'Algérois: Nouvelles données géochronologiques. Géologie Méditerranéenne, 4(4), 291-297. https://doi.org/10.3406/geolm.1977.1010

Besse, J., \& Courtillot, V. (2002). Apparent and true polar wander and the geometry of the geomagnetic field over the last 200 Myr. Journal of Geophysical Research, 107(B11), EPM 6-1-EPM 6-31. https://doi.org/10.1029/2000JB000050

Bouchez, J. L. (2000). Anisotropie de susceptibilité et fabrique des granites. Comptes Rendus Académie Sciences, Paris, Earth Planetary Science, 330, 1-14.

Cañón-Tapia, E., Walker, G. P. L., \& Herrero-Bervera, E. (1995). Magnetic fabric and flow direction in basaltic Pahoehoe lava of Xitle Volcano, Mexico. Journal of Volcanology and Geothermal Research, 65(3-4), 249-263. https://doi.org/10.1016/0377-0273(94)00110-3

Channell, J. E. T., Oldow, J. S., Catalano, R., \& D'Argenio, B. (1990). Paleomagnetically determined rotations in the western Sicilian fold and thrust belt. Tectonics, 9(4), 641-660. https://doi.org/10.1029/TC009i004p00641

Deenen, M. H. L., Langereis, C. G., van Hinsbergen, D. J. J., \& Biggin, A. J. (2011). Geomagnetic secular variation and the statistics of palaeomagnetic directions. Geophysical Journal International, 186(2), 509-520. https://doi.org/10.1111/j.1365-246X.2011.05050.x

Demarest, H. H. (1983). Error analysis for the determination of tectonic rotation from paleomagnetic data. Journal of Geophysical Research, 88(B5), 4321-4328. https://doi.org/10.1029/JB088iB05p04321

Derder, M.E.M., Henry, B., Amenna, M., Bayou, B., Maouche, S., Besse, J., et al. (2011), Tectonic evolution of the active "Cheliff” Basin (Northern Algeria) from paleomagnetic and magnetic fabric investigations. In New frontiers in tectonic research at the midst of plate convergence Intech Publisher book, pp: 3-26, Intech Publisher, ISBN 978-953-307-594-5.

Derder, M. E. M., Henry, B., Maouche, S., Amenna, M., Bayou, B., Besse, J., et al. (2013). Transpressive tectonics along a major E-W crusta structure on the Algerian continental margin: Block rotation revealed by paleomagnetic investigations. Tectonophysics, 593, 183-192. https://doi.org/10.1016/j.tecto.2013.03.007

Derder, M. E. M., Maouche, S., Robion, P., Henry, B., Amenna, M., Bayou, B., et al. (2018). Neotectonic deformation model of the northern Algeria from paleomagnetic data: Preliminary results from Northwestern areas. In N. Sundararajan, et al. (Eds.), On significant applications of geophysical methods, Advances in Science, Technology \& Innovation, (pp. 39-41). doi: https://doi.org/10.1007/978-3-030-0165628

Dewey, J. F., Helman, M. L., Turco, E., Hutton, D. H. W., \& Knott, S. D. (1989). Kinematics of the western Mediterranean. In M. P. Coward, D. Dietrich, \& R. G. Park (Eds.), Alpine tectonics, (pp. 265-283). London: Geol. Soc.

Di Chiara, A., Speranza, F., \& Porreca, M. (2012). Paleomagnetic secular variation at the Azores during the last 3 ka. Journal of Geophysical Research, 117, B07101(B7). https://doi.org/10.1029/2012JB009285

Domzig, A., Yelles, K., Le Roy, C., Déverchère, J., Bouillin, J. P., Bracène, R., et al. (2006). Searching for the Africa-Eurasia Miocene boundary offshore western Algeria MARADJA'03 cruise. Comptes Rendus Geoscience, 338(1-2), 80-91. https://doi.org/10.1016/j. crte.2005.11.009

Durand, D. M. (1969). Mise au point sur la structure du Nord Est de la Berbérie. Bulletin Services Carte Géologie Algérie, 39, 89-131.

Enkin, R. J., \& Watson, G. S. (1996). Statistical analysis of palaeomagnetic inclination data. Geophysical Journal International, 126(2), 495-504. https://doi.org/10.1111/j.1365-246X.1996.tb05305.x

Fisher, R.A. (1953), Dispersion on a sphere. Proc. Roy. Soc., London A217, 295-305.

Gattacceca, J., \& Rochette, P. (2002). Pseudopaleosecular variation due to remanence anisotropy in a pyroclastic flow succession. Geophysical Research Letters, 29, 1286.

Georgiev, N., Henry, B., Jordanova, N., Jordanova, D., \& Naydenov, K. (2014). Emplacement and fabric-forming conditions of plutons from structural and magnetic fabric analysis: Case study of the Plana massif (central Bulgaria). Tectonophysics, 629, 138-154. https://doi.org/ 10.1016/j.tecto.2014.02.018

Girod, M., \& N. Girod, N. (1977). Contribution de la pétrologie à la connaissance de l'évolution de la Méditerranée occidentale depuis l'Oligocène. Bull. Société Géologique de France, XIX(7), 481-488.

Guemache, M. (2010), Evolution géodynamique des bassins sismogènes de l'Algérois (Algérie): Approche pluridisciplinaire (méthodes géologiques et géophysiques, $\mathrm{PhD}$ thesis, University Houari Boumediene University of Sciences and Technology, $294 \mathrm{pp}$.

Henry B. (1980), Contribution à l'étude des propriétés magnétiques de roches magmatiques des Alpes: Conséquences structurales, régionales et générales. Thesis, Paris, 528 pp.

Henry, B., Derder, M. E. M., Maouche, S., Nouar, O., Amenna, M., Bayou, B., \& Ouabadi, A. (2019). An overview of the plutons magnetic fabric studies in the Hoggar shield: Evolution of the major shear zones during the Pan-African. In A. Bendaoud, Z. Hamimi, M. Hamoudi, S. Djemal, \& B. Zoheir (Eds.), Geology of the Arab world. An overview, (pp. 149-166). Springer. doi: https://doi.org/10.1007/ 978-3-319-96794-3_4 
Henry, B., Homberg, C., Mroueh, M., Hamdan, W., \& Higazi, F. (2010). Rotations in Lebanon inferred from new palaeomagnetic data and implications for the evolution of the Dead Sea Transform system. In C. Homberg, \& M. Bachmann (Eds.), Evolution of the Levant margin and western Arabia Platform since the Mesozoic, (Vol. 341, pp. 269-285). London, 2010: Geol. Soc. Special Pub. https://doi.org/10.1144/ SP341.13

Henry, B., Plenier, G., \& Camps, P. (2003). Post-emplacement titlting of lava flows inferred from magnetic fabric study: The example of Oligocene lavas in the Jeanne d'Arc Peninsula (Kerguelen Islands). Journal of Volcanology and Geothermal Research, 127(1-2), 153-164. https://doi.org/10.1016/S0377-0273(03)00198-7

Hernandez J., De Larouzière, F.D., Bolze, J., \& Bordet, P. (1987), Le magmatisme néogène bético-rifain et le couloir de décrochement transAlboran. Bull. Soc. géol. France, (8), III, 257-267.

Hernandez, J., \& Lepvrier, C. (1979), Le volcanisme calco-alcalin miocène de la région d'Alger (Alger). Bull. Soc. géol. France (7), XXI, 73-

Hext, G. (1963). The estimation of second-order tensors, with related tests and designs. Biometrika, 50(3-4), 353-373. https://doi.org/ 10.1093/biomet/50.3-4.353

Hrouda, F., \& Ježek, J. (2017). Role of single-domain magnetic particles in creation of inverse magnetic fabrics in volcanic rocks: A mathematical model study. Studia Geophysica et Geodaetica, 61(1), 145-161. https://doi.org/10.1007/s11200-015-0675-6

Jelinek, V. (1978). Statistical processing of magnetic susceptibility measured in groups of specimens. Studia Geophysica et Geodetica, 22(1), 50-62. https://doi.org/10.1007/BF01613632

Jelinek, V. (1981). Characterization of the magnetic fabric of rocks. Tectonophysics, 79, 63-67.

Kirschvink, J. L. (1980). The least-squares line and plane and the analysis of palaeomagnetic data. Geophysical Journal of the Royal Astronomical Society, 62(3), 699-718. https://doi.org/10.1111/j.1365-246X.1980.tb02601.x

Krijgsman, W., \& Tauxe, L. (2004). Shallow bias in Mediterranean paleomagnetic directions caused by inclination error. Earth and Planetary Science Letters, 222(2), 685-695. https://doi.org/10.1016/j.epsl.2004.03.007

Le Pichon, X., Bergerat, F., \& M.J. Roulet (1988), Plate kinematics and tectonic leading to Alpine belt formation: A new analysis, Processes in continental lithospheric deformation. Geol. Soc. Amer. Spec. Paper, 111-131.

Leprêtre, A., Klingelhoefer, F., Graindorge, D., Schnurle, P., Beslier, M. O., Yelles, K., et al. (2013). Multiphased tectonic evolution of the Central Algerian margin from combined wide-angle and reglection seismic data off Tipaza, Algeria. Journal Geophysical Research, 118(8), 3899-3916. https://doi.org/10.1002/jgrb.50318

Lepvrier, C., Magne, J., \& Sigal, J. (1970), Données stratigraphiques et structurales sur les formations telliennes d'une partie du tell septentrional (secteur compris entre Cherchell, Miliana, El Asnam et Tenes), Algérie. Bull. Soc. géol. France, XII, 794-804.

Mandl, G. (1987). Tectonic deformation by rotating parallel faults: The "bookshelf" mechanism. Tectonophysics, 141(4), 277-316. https:// doi.org/10.1016/0040-1951(87)90205-8

Maouche, S., \& Harbi, A. (2018). The active faults of the Mitidja basin (North Central Algeria): What does the seismic history of the region tell us? Euro-Mediterranean Journal Environmental Integration, 3(1), 1-11. https://doi.org/10.1007/s41207-018-0061-1

Maouche, S., Meghraoui, M., Morhange, C., Belabbes, S., Bouhadad, Y., \& Hadoum, H. (2011). Active coastal thrusting and folding, and uplift rate of the Sahel Anticline and Zemmouri earthquake area (Tell Atlas, Algeria). Tectonophysics, 509(1-2), 69-80. https://doi.org/ 10.1016/j.tecto.2011.06.003

Mauffret, A. (2007). The Northwestern (Maghreb) boundary of the Nubia (Africa) plate. Tectonophysics, 429(1-2), 21-44. https://doi.org/ 10.1016/j.tecto.2006.09.007

Mauffret, A., El Robrini, M., \& Gennesseaux, M. (1987), Indice de la compression récente en mer Méditerranée: Un bassin losangique sur la marge algérienne. Bull. Soc. géol. France (8), III, 6, 1195-1206.

Mazzoli, S., \& Helman, M. (1994). Neogene patterns of relative plate motion for Africa- Europe: Some implications for recent central Mediterranean tectonics. Geologische Rundschau, 83, 464-468.

Meghraoui, M. (1988), Géologie des zones sismiques du nord de l'Algérie (Paléosismologie, Tectonique active et synthèse sismotectonique), $\mathrm{PhD}$ thesis, Université de Paris-Sud, Centre d'Orsay, France, $356 \mathrm{pp}$.

Meghraoui, M. (1991). Blind reverse faulting system associated with the Mont Chenoua-Tipasa earthquake of 29 October 1989 (northcentral Algeria). Terra Nova, 3(1), 84-92. https://doi.org/10.1111/j.1365-3121.1991.tb00847.x

Meghraoui, M. (2018). Earthquake faulting and their implications for the seismic hazard assessment along the plate boundary in North Africa. In A. Kallel, et al. (Eds.), Recent advances in environmental science from the Euro-Mediterranean and surrounding regions, Advances in Science, Technology and Innovation. doi: https://doi.org/10.1007/978-3-319-70548-4_15

Meghraoui, M., Cisternas, A., \& Philip, H. (1986). Seismotectonics of the lower Cheliff basin: structural background of the El Asnam (Algeria) earthquake. Tectonics, 5(6), 809-836. https://doi.org/10.1029/TC005i006p00809

Meghraoui, M., \& Doumaz, F. (1996). Earthquake-induced flooding and paleoseismicity of the El Asnam (Algeria) fault-related fold Journal of Geophysical Research, 101(B8), 17617-17644. https://doi.org/10.1029/96JB00650

Meghraoui, M., Maouche, S., Chemaa, B., Cakir, Z., Aoudia, A., Harbi, A., et al. (2004). Coastal uplift and thrust faulting associated with the $\mathrm{Mw}=6.8$ Zemmouri (Algeria) earthquake of 21 May, 2003. Geophysical Research Letters, 31(19), L19605. https://doi.org/10.1029/ 2004GL020466

Meghraoui, M., Morel, J.L., Andrieux, J., \& Dahmani, M. (1996), Tectonique plio-quaternaire de la chaîne tello-rifaine et de la mer d'Alboran. Une zone complexe de convergence continent-continent. Bull. Soc. géol. France,167, 1, 141-157.

Meghraoui, M., \& Pondrelli, S. (2012). Active faulting and transpression tectonics along the plate boundary in North Africa. Annals of Geophysics, 55(5), 2012. https://doi.org/10.4401/ag-4970

Morel, J. L., \& Meghraoui, M. (1996). Goringe-Alboran-Tell tectonic zone; a transpression system along the Africa-Eurasia plate boundary. Geology, 24(8), 755-758. https://doi.org/10.1130/0091-7613(1996)024<0755:GATTZA>2.3.CO;2

Nocquet, J. M. (2012). Present-day kinematics of the Mediterranean: A comprehensive overview of GPS results. Tectonophysics, 579, 220-242. https://doi.org/10.1016/j.tecto.2012.03.037

Nocquet, J. M., \& Calais, E. (2004). Geodetic measurements of crustal deformation in the western Mediterranean and Europe. Pure and Applied Geophysics, 161(3), 661-681, 0033 - 4553/04/030661 - 21. https://doi.org/10.1007/s00024-003-2468-Z

Nouar, O., Henry, B., Liégeois, J. P., Derder, M. E. M., Bayou, B., Bruguier, O., et al. (2011). Eburnean and Pan-African granitoids and the Raghane mega-shear zone evolution: Image analysis, U-Pb zircon age and AMS study in the Arokam Ténéré (Tuareg shield, Algeria). Journal of African Earth Sciences, 60, 133-152. https://doi.org/10.1016/j.jafrearsci.2011.02.007

Ousadou, F., Dorbath, L., Ayadi, A., Dorbath, C., \& Gharbi, S. (2014). Stress field variations along the Maghreb region derived from inversion of major seismic crisis fault plane solutions. Tectonophysics, 632, 261-280. https://doi.org/10.1016/j.tecto.2014.06.017 
Ouyed, M., Meghraoui, M., Cisternas, A., Deschamp, A., Dorel, J., Frechet, F., et al. (1981). Seismotectonics of the El Asnam earthquake. Nature, 292(5818), 26-31. https://doi.org/10.1038/292026a0

Plénier, G., Camps, P., Henry, B., \& Ildefonse, B. (2005). Determination of flow directions by combining AMS and thin-section analyses for Oligocene volcanism in the Kerguelen Archipelago (Southern Indian Ocean). Geophysical Journal International, 160, 63-78.

Ratzov, G., Cattaneo, A., Babonneau, N., Déverchère, J., Yelles, K., Bracene, R., \& Courboulex, F. (2015). Holocene turbidites record earthquake supercycles at a slow-rate plate boundary. Geology, 43(4), 331-334. https://doi.org/10.1130/G36170.1

Ricou, L. E. (1994). Tethys reconstructed: Plates continental fragments and their boundaries since 260 Ma from Central America to Southeastern Asia. Geodinamica Acta, 7(4), 169-218. https://doi.org/10.1080/09853111.1994.11105266

Rosenbaum, G., Lister, G. S., \& Duboz, C. (2002). Relative motions of Africa, Iberia and Europe during Alpine orogeny. Tectonophysics, 359(1-2), 117-129. https://doi.org/10.1016/S0040-1951(02)00442-0

Schult, A. (1976). Paleomagnetism of Cretaceous and Jurassic volcanic rocks in western Sicily. Earth and Planetary Science Letters, 31(3), 454-457. https://doi.org/10.1016/0012-821X(76)90126-6

Serpelloni, E.,. G., Vannucci, S., Pondrelli, A., Argnani, G., Casula, M., Anzidei, P., et al. (2007). Kinematics of the Western Africa-Eurasia plate boundary from focal mechanisms and GPS data. Geophysical Journal International, 169(3), 1180-1200. https://doi.org/10.1111/ j.1365-246X.2007.03367.x

Silva, P. F., Henry, B., Marques, F. O., Hildenbrand, A., Lopes, A., Madureira, P., et al. (2018). Volcano-tectonic framework of a linear volcanic ridge (Faial-Pico ridge, Azores Archipelago) assessed by paleomagnetic studies. Journal Volcanology Geothermal Rereach, 352, 78-91. https://doi.org/10.1016/j.jvolgeores.2018.01.005

Smith, B., Derder, M. E. M., Henry, B., Bayou, B., Amenna, M., Djellit, H., et al. (2007). Relative importance of the Hercynian and postJurassic tectonic phases in the Saharan platform: A palaeomagnetic study of Jurassic sills in the Reggane basin (Algeria). Geophysical Journal International, 167, 380-396.

Soumaya, A., Ben Ayed, N., Rajabi, M., Meghraoui, M., Delvaux, D., Kadri, A., et al. (2018). Active faulting geometry and stress pattern near complex strike-slip systems along the Maghreb Region: Constraints on active convergence in the Western Mediterranean. Tectonics, 37(9), 3148-3173. https://doi.org/10.1029/2018TC004983

Speranza, F., Hernandez-Moreno, C., Avellone, G., Morticelli, M. G., Agate, M., Sulli, A., \& Di Stefano, E. (2018). Understanding paleomagnetic rotations in Sicily: Thrust versus strike-slip tectonics. Tectonics, 37(4), 1138-1158. https://doi.org/10.1002/2017TC004815

Thomas, G. (1985), Géodynamique d'un bassin intramontagneux: Le bassin du bas Chéliff occidental (Algérie) durant le Mio-PlioQuaternaire, PhD thesis, Pau University, France

Thompson, J., Guillaume, A., \& Daly, L. (1986). Paleomagnetism of the Permian volcanic rocks of Moissey (French Jura): Implications for the paleofield and tectonic evolution. Geophysical Journal of the Royal Astronomical Society, 86(1), 103-117. https://doi.org/10.1111/ j.1365-246X.1986.tb01075.x

Tomezzoli, R. N., McDonald, W. D., \& Tickyj, H. (2003). Composite magnetic fabrics and S-C structure in granitic gneiss of Cerro de los Viejos, La Pampa province, Argentina. Journal Structural Geology, 25(2), 159-169. https://doi.org/10.1016/S0191-8141(02)00030-5

Wernicke, B., \& Burchfiel, B. (1982). Modes of extensional tectonics. Journal of Structural Geology, 4(2), 105-115. https://doi.org/10.1016/ 0191-8141(82)90021-9

Zijderveld, J. D. A. (1967). AC demagnetization of rocks: Analysis of results. In D. W. Collinson, K. M. Creer, \& S. K. Runcorn (Eds.), Method in paleomagnetism, (pp. 254-286). Amsterdam: Elsevier. 